\title{
Gradhiva
}

\section{Races (1930). À propos d'un ouvrage oublié de Jean Brunhes}

Races (1930). About a Forgotten Book by Jean Brunhes

\section{Teresa Castro}

\section{(2) OpenEdition \\ 1 Journals}

\section{Édition électronique}

URL : http://journals.openedition.org/gradhiva/3540

DOI : 10.4000 /gradhiva. 3540

ISSN : 1760-849X

\section{Éditeur}

Musée du quai Branly Jacques Chirac

\section{Édition imprimée}

Date de publication : 23 mai 2018

Pagination : 144-175

ISBN : 978-2-35744-110-1

ISSN : 0764-8928

\section{Référence électronique}

Teresa Castro, «Races (1930). À propos d'un ouvrage oublié de Jean Brunhes », Gradhiva [En ligne], 27 | 2018, mis en ligne le 23 mai 2020, consulté le 06 janvier 2021. URL : http://

journals.openedition.org/gradhiva/3540; DOI : https://doi.org/10.4000/gradhiva.3540

\section{(C) musée du quai Branly}


d6 -

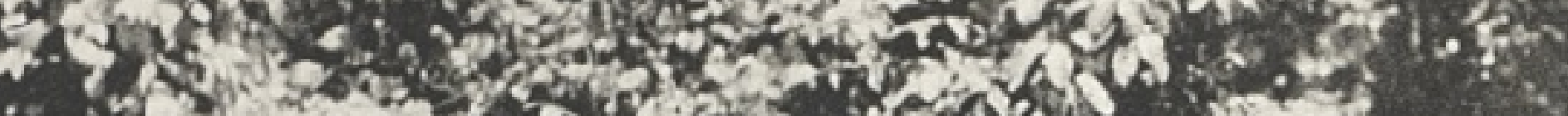

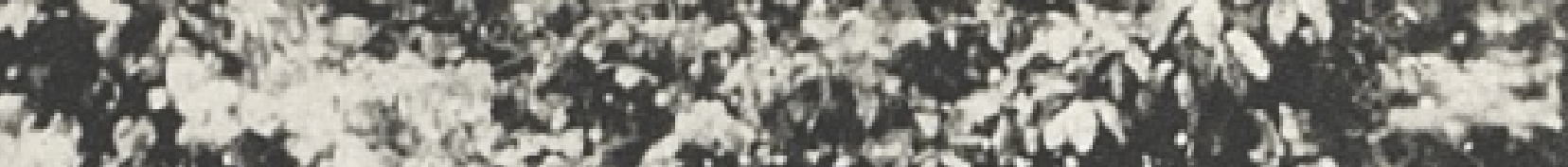

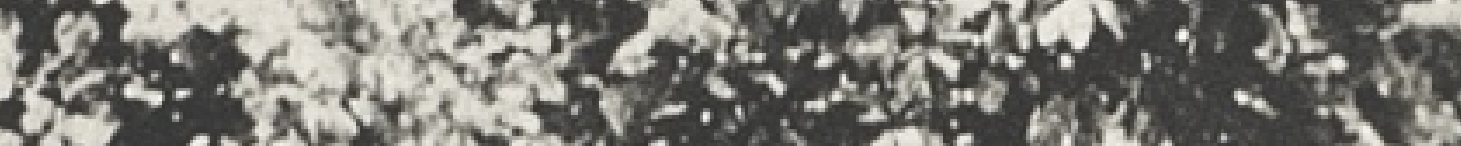

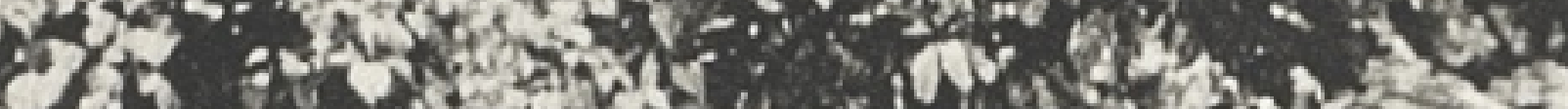

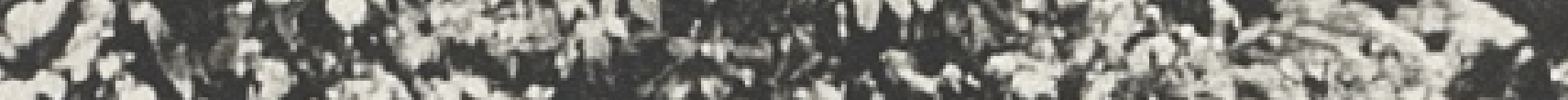

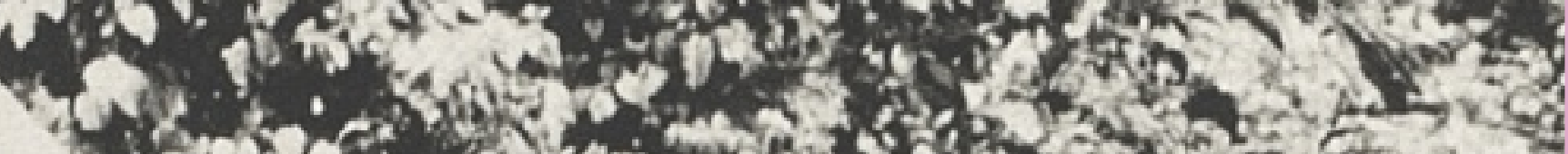
4.

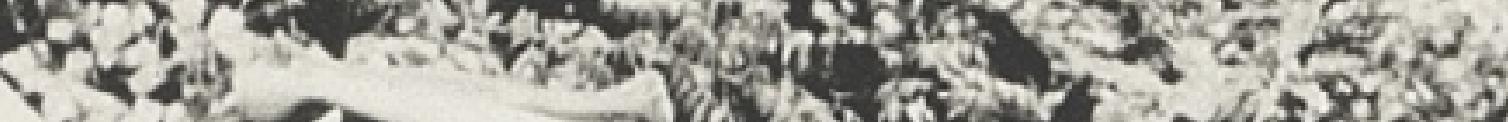

(6)

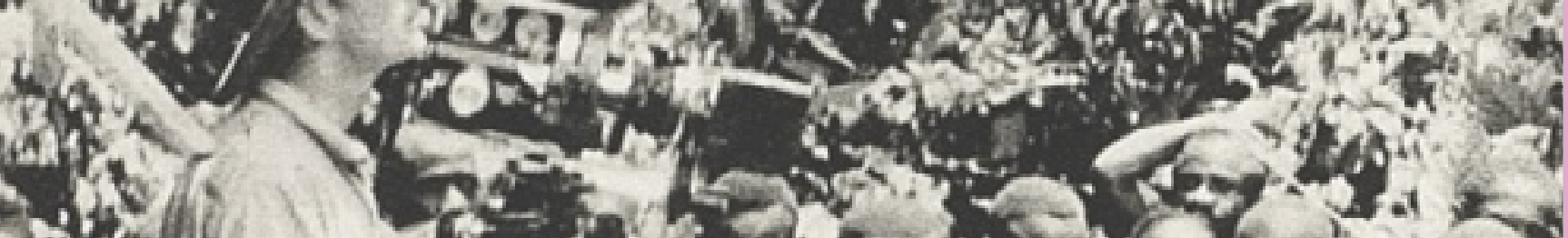
ats

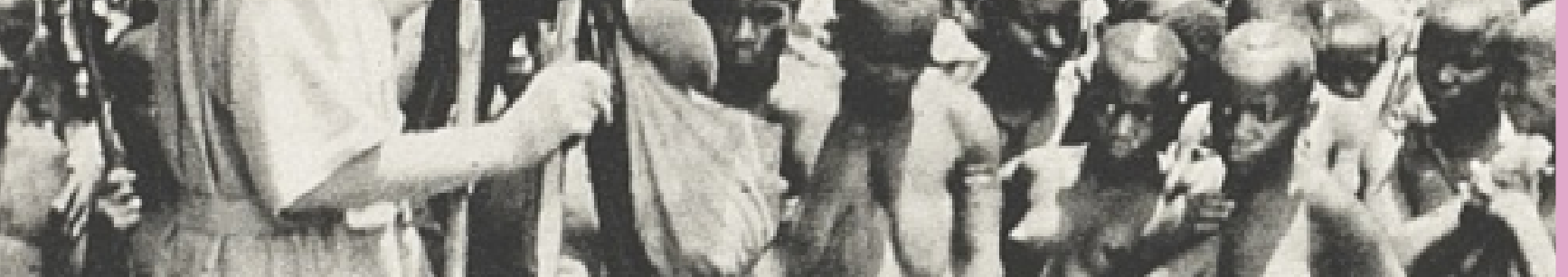

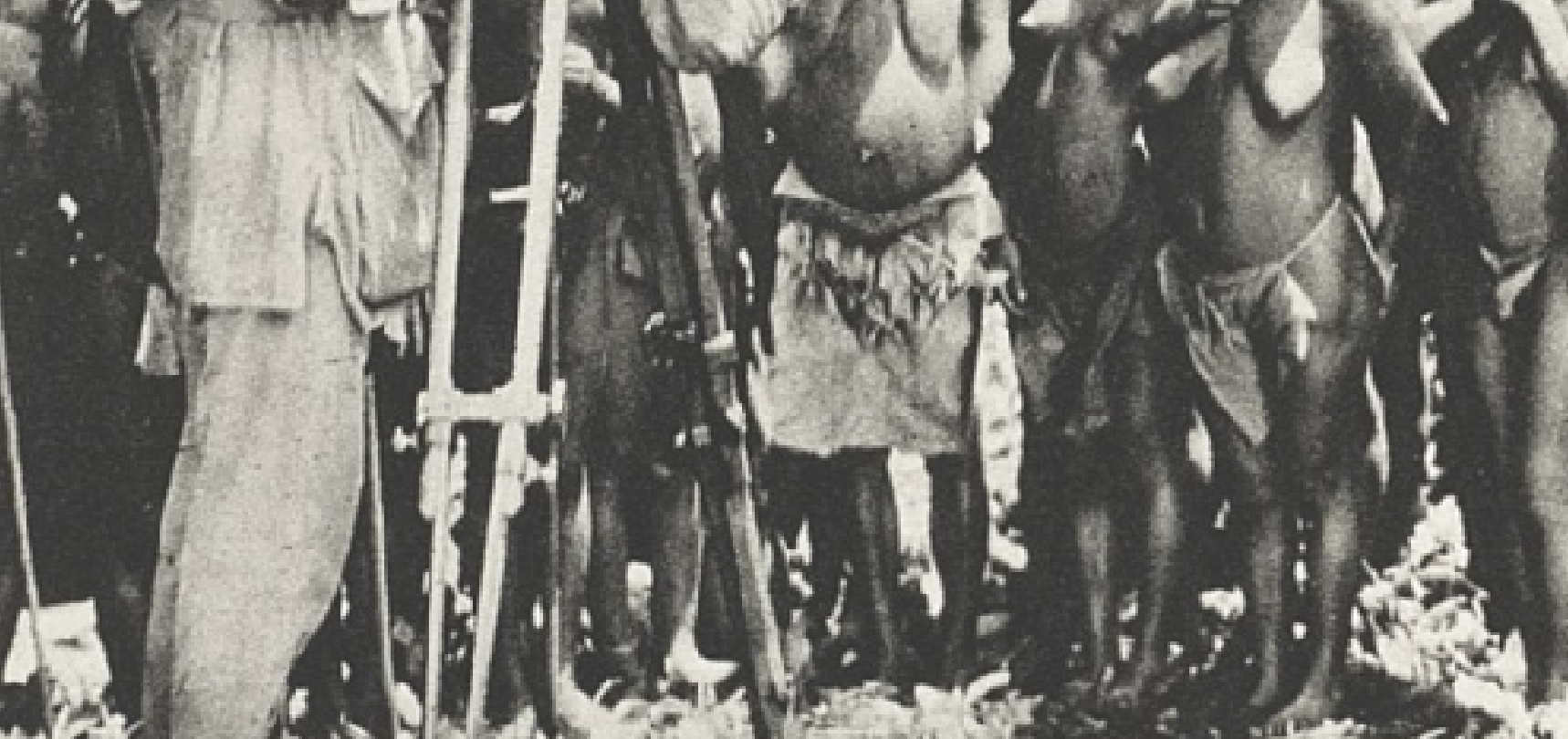

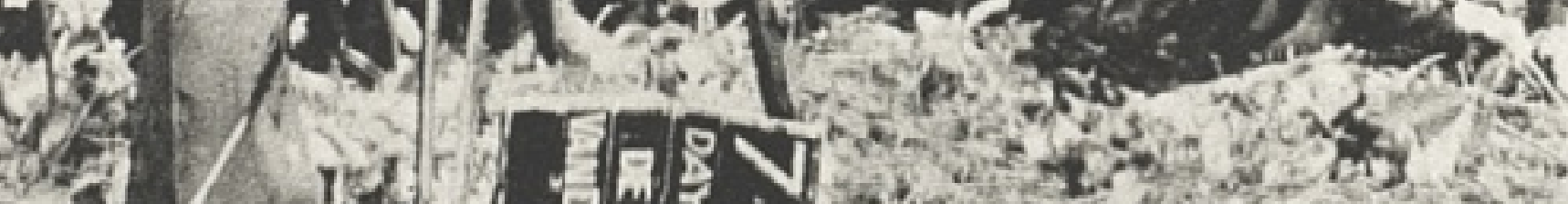

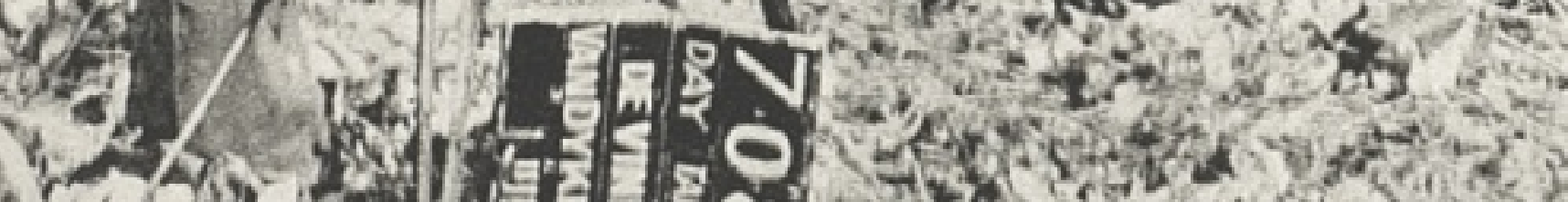

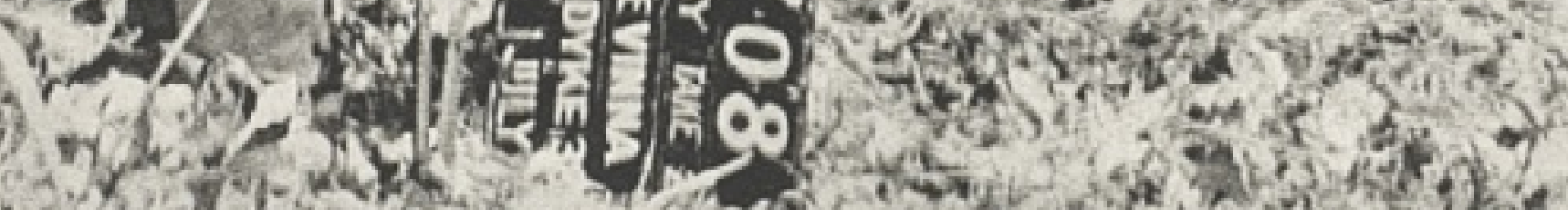

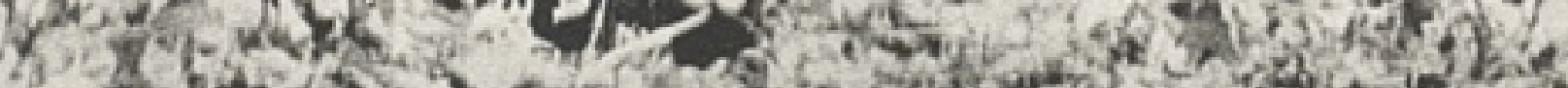

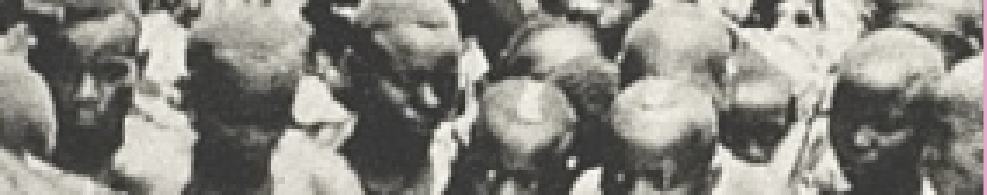

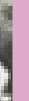


Races (1930)

À propos d'un ouvrage oublié de Jean Brunhes

par Teresa Castro

Publié en 1930 dans la collection «Images du monde », que Florent Fels dirige chez Firmin-Didot, l'ouvrage Races du géographe Jean Brunhes constitue ce qu'on pourrait appeler un atlas de vulgarisation. Illustré par un matériel photographique hétérogène (clichés du xIx ${ }^{e}$ siècle, images d'agences photographiques, photographies de tournage de films hollywoodiens, etc.), le livre est le support d'un discours paradoxal sur la diversité humaine. Cet objet, fruit d'une culture visuelle à cheval entre deux disciplines (la géographie humaine et l'anthropologie) et deux sphères d'activité (la vulgarisation scientifique et l'édition artistique), produit un discours que nous analyserons ici en l'inscrivant dans le paysage scientifique et éditorial de l'époque. Une attention particulière est accordée aux liens entre Races et un autre projet auquel le géographe fut intimement lié: la collection d'images des Archives de la Planète (1912-1931) d'Albert Kahn. 


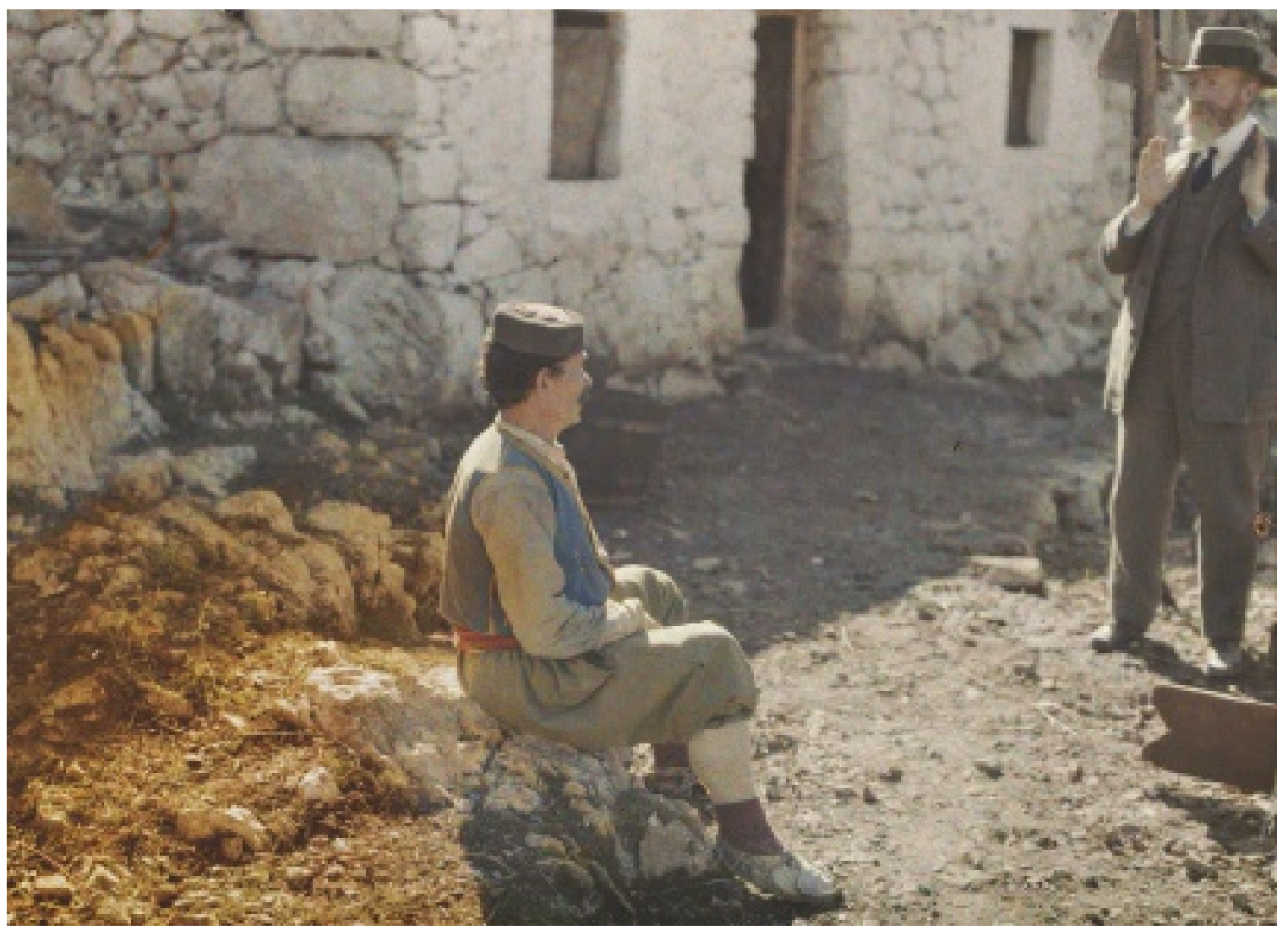

fig. 1

Auguste Léon, Un paysan

assis : le bonnet avec les

initiales (Kapa), Monténégro,

Cettigné, 23 octobre 1913.

Boulogne-Billancourt,

musée départemental

Albert Kahn, collection

Archives de la Planète,

inv. A 2931.

Jean Brunhes, visible à

droite de l'image, donne

des indications de

cadrage. 
Dans les pages de la revue Documents, Michel Leiris s'extasie, en 1930, devant un ouvrage illustré venant alors tout juste de paraître:

En cette Europe chaque jour plus sordide dans laquelle nous vivons, l'exotisme exerce un attrait de plus en plus violent sur un certain nombre d'esprits [...]. Ce prestige puissant que possède l'exotisme (puissant au point de se transformer pour quelques-uns en une réelle nostalgie) explique dans une large mesure la vogue dont jouit actuellement l'ethnographie. Mais il manquait encore en France un livre qui présentât au grand public un choix de documents purement ethnographique et pas simplement une série d'œuvres d'art. Le dernier ouvrage publié par le grand savant Jean Brunhes, qui vient de disparaître, remplit cette lacune. II s'agit, en effet, d'un recueil de reproductions excellentes tirées de diverses collections et représentant quelques types choisis des principales races du monde, photographiées dans une de leurs occupations caractéristiques. (Leiris 1930: 375)

Le livre en question s'intitule Races: publié en 1930 dans la collection «Images du monde » de l'éditeur Firmin-Didot, il constitue, comme le signale Leiris, le dernier opus du géographe Jean Brunhes, disparu prématurément en août 1930. L'ouvrage est ce qu'on pourrait appeler un atlas de vulgarisation illustré par un matériel photographique extrêmement hétérogène, combinant clichés du XIX ${ }^{e}$ siècle issus de la collection du laboratoire d'anthropologie du Muséum, images d'agences photographiques ou de presse, photographies de tournage de films hollywoodiens, documents originalement publiés par des instances administratives françaises et étrangères, etc. Se situant aux frontières de ces deux sciences encore voisines que sont la géographie humaine et l'ethnologie, cet étonnant recueil photographique est le support d'un discours sur la diversité humaine. En ce sens, il n'est pas sans rappeler un autre projet, jamais mentionné dans le livre, auquel le géographe fut pourtant intimement lié: les «Archives de la Planète». Cette collection d'autochromes et de films fut créée en 1912 par le financier et mécène français Albert Kahn, qui s'apprête, au moment de la publication de l'ouvrage de Brunhes, à cesser ses activités. Mon propos ici est d'interroger, du point de vue de la culture visuelle, cet objet singulier, à cheval entre deux disciplines (la géographie humaine et l'anthropologie) et deux sphères d'activité (la vulgarisation scientifique et l'édition dite «artistique»). Pour ce faire, j'inscris Races dans le paysage scientifique et éditorial de son époque, j'en examine les images photographiques et questionne, pour finir, le discours profondément paradoxal que cet atlas véhicule sur la diversité humaine, à un moment où la notion de race est mise au service d'idéologies de plus en plus virulentes, pour justifier nationalismes ethnocentriques et/ou exploitation coloniale. Bien que la perspective choisie ne soit pas celle de l'histoire des disciplines géographique et/ou anthropologique, cette enquête évoque rapidement les rapports entre la géographie humaine et l'ethnographie dans le contexte intellectuel français du début du $x x^{e}$ siècle. Il y est aussi question de l'histoire de l'édition photographique en France. Enfin, j'ai choisi d'explorer l'expérience de Brunhes dans les Archives de la Planète, car si celle-ci ne constitue qu'un volet de son activité de géographe, elle permet toutefois d'inscrire plus largement Races dans la culture visuelle de son temps. 
1. Cité par Emmanuel de Margerie: lettre à Jean Brunhes, 26 janvier 1912 Archives Jean Brunhes.

\section{Jean Brunhes, la géographie humaine et l'ethnologie}

Mais qui était donc Jean Brunhes? Géographe, catholique social engagé, professeur au Collège de France et membre de l'Institut, Brunhes est un éminent représentant de l'élite intellectuelle de la Troisième République. Fils d'universitaire, né à Toulouse en 1869, il étudie à l'École normale supérieure de Paris avant de s'orienter, sur les conseils de son maître Paul Vidal de La Blache, vers une carrière de géographe. En 1902, il soutient une thèse sur l'irrigation puis publie en 1910 son œuvre capitale, maintes fois rééditée, La Géographie humaine. Ce mémoire atteste l'influence du géographe et ethnographe allemand Friedrich Ratzel, l'un des fondateurs de l'«anthropogéographie », dont Brunhes a suivi les séminaires en Allemagne (Robic 2014 :18). La géographie humaine en France s'intéresse aux relations des hommes à leur milieu, mais oppose au déterminisme de Ratzel une perspective interactionniste, qui met l'accent sur les choix humains et les faits culturels. Si Vidal de La Blache parle de «possibilisme», l'approche de Brunhes pourrait presque être qualifiée de culturaliste avant la lettre. Autre point essentiel: la sensibilité du géographe au visuel, et en particulier à la photographie, qu'il pratique depuis son adolescence et qu'il ne cessera de promouvoir tout au long de sa vie. Si cette technique semble «consacrée dans la géographie universitaire des années 1910 » (Robic 1993a: 59), Brunhes est un véritable "géo-photo-graphe » accordant un rôle déterminant - à la fois descriptif, démonstratif et pédagogique - à l'image photographique (Robic 1993b; Mendibil 1993a).

En 1912, Brunhes, sur invitation du banquier Albert Kahn, devient le directeur scientifique des Archives de la Planète. Entre 1909 et 1931, sont rassemblés dans cette collection soixante-douze mille autochromes, quatre mille photographies stéréoscopiques et plus de cent heures de films, réalisés par une dizaine d'opérateurs en France et dans une cinquantaine d'autres pays. Le souhait de Kahn (qui emploie sa vaste fortune à des œuvres philanthropiques) est de témoigner, sur le plan politique et moral, d'un monde à la merci des changements rapides et irréversibles entraînés par la modernisation, comme l'illustrent ses propos sur sa volonté d'utiliser «la photographie stéréoscopique, les projections [d'autochromes], le cinématographe surtout [...] afin de fixer une fois pour toutes des aspects, des pratiques et des modes de l'activité humaine dont la disparition fatale n'est plus qu'une question de temps ${ }^{1}$ ». II ne s'agit en aucun cas d'entretenir un regard nostalgique ou exotique, mais de soutenir, par le biais de cette documentation proliférante, un projet social et politique complexe, appuyé sur les multiples fondations de Kahn et se donnant pour mission de contribuer à la paix mondiale, à la souveraineté des nations et à une forme d'internationalisme élitiste. Si le projet du banquier ne coïncide pas en tout point avec la vision sans doute plus utilitariste que Brunhes aura des Archives, les deux hommes partagent toutefois des valeurs essentielles. Avec sa femme Henriette Hoskier, Brunhes évolue dans le milieu du catholicisme social et fréquente les réseaux réformateurs: il est donc sensible à l'engagement humanitaire et humaniste de Kahn. Par ailleurs, la croyance et la confiance de Kahn en l'objectivité de la photographie et du cinéma résonnent certainement chez Brunhes, pour qui les autochromes sont comme «un atelier de preuves décisives » ou «un arsenal de révélations » (Brunhes, cité dans Robic 1993b: 109). 


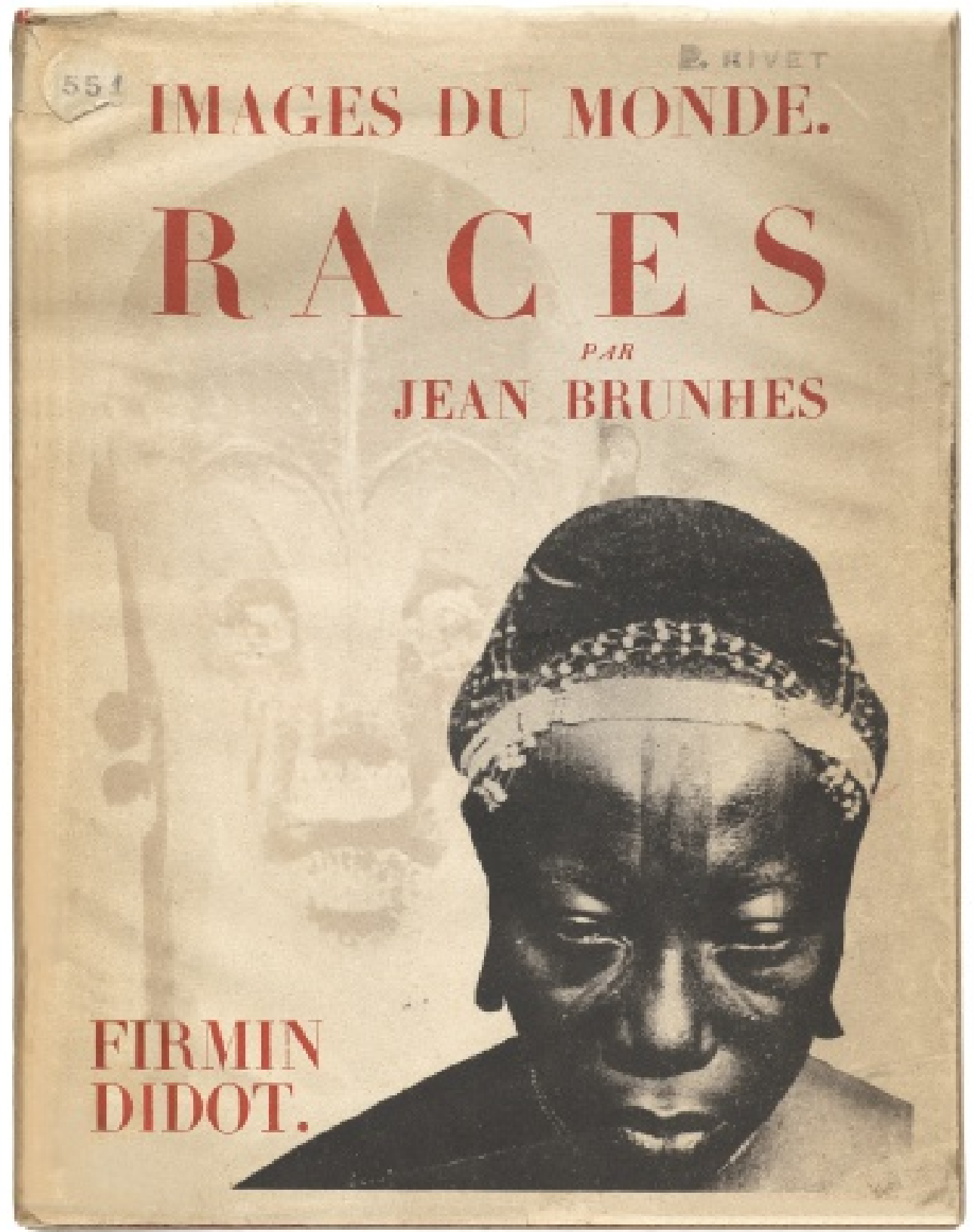

fig. 2

Couverture de l'album

de Jean Brunhes, Races

paru en 1930 aux éditions

Firmin-Didot, Paris. 
2. Sur la géographie au Collège de France, voir Orain et Robic 2017. Sur les débats conduisant à l'élection de Brunhes au Collège de France, voir Deprest 2017: 209-218.

3. Dans ce texte, Mauss consacre plusieurs pages à la discipline géographique se sentant en particulier obligé de spécifier sa " position à l'égard des méthodes que pratique la discipline spéciale qui a pris le nom d'anthropogéographie " (Mauss 1906: 41).

4. À propos des liens entre géographie et ethnologie, voir le numéro spécial "Ethnologues et géographes " de la revue Ethnologie française 34 (4), en particulier Robic 2004 et Blanckaert 2004.

Signalons que si les Archives de la Planète proscrivent la collecte d'objets (activité à laquelle ne s'intéresse pas non plus Jean Brunhes), Mariel Jean-Brunhes est une collecteuse importante, réalisant dès les années 1950 et pour le compte du musée national des Arts et des Traditions populaires plusieurs missions. Comme l'écrira Georges-Henri Rivière en 1964, "notre musée-laboratoire d'ethnologie française lui est redevable d'une
Détail décisif, au moment où Brunhes prend son poste de directeur des Archives, la chaire de géographie humaine est créée au Collège de France et Brunhes en devient le fier titulaire jusqu'à sa mort ${ }^{2}$, ce qui lui permet de poursuivre sa carrière en France, après seize années passées en Suisse. II s'agit par ailleurs d'un moment crucial pour l'institutionnalisation de cette nouvelle discipline, dont l'un des enjeux est alors de se démarquer de l'ethnographie, décrite comme «voisine» par Brunhes lui-même (1913: 29). Le géographe semble conscient de la nécessité de tracer des frontières claires entre ces deux domaines, d'autant plus que le projet des Archives de la Planète, animé par l'urgence de garder des traces des modes de vie voués à disparaître, le rapproche dangereusement du terrain ethnographique. Dans La Géographie humaine, Brunhes inclut un sous-chapitre intitulé «Géographie humaine et géographie ethnographique", visant à "distinguer très nettement" ces deux disciplines (Brunhes 1910: 619). Son argument consiste à défendre que les «faits fondamentaux» de la géographie humaine sont pour l'ethnographie des "faits accessoires" et vice-versa. Lors de la parution du livre, l'ethnologue et folkloriste français Arnold Van Gennep lui reproche toutefois de venir occuper le terrain de l'ethnographie. En 1913, Brunhes publie dans les pages de l'Ethnographie l'essai «Ethnographie et géographie humaine», où il essaie à nouveau de négocier au mieux le partage disciplinaire, tout en plaidant la complémentarité et la nécessité pour géographes et ethnographes de travailler ensemble. Ce texte réflexif, où il est question du projet des Archives de la Planète (voir infra), fait partie, avec une étude pionnière de Marcel Mauss - «Essai sur les variations saisonnières des sociétés Eskimos. Étude de morphologie sociale" $(1906)^{3}-$, des premières tentatives de confronter les deux disciplines. Dans leur sillage, d'autres textes suivront, signés par le géographe Jules Sion (1937), l'ethnologue et historien André Leroi-Gourhan (1948), le géographe Max Sorre (1957), ou encore la géographe (fille et collaboratrice de Brunhes) Mariel Jean-Brunhes Delamarre (1968) ${ }^{4}$. De Van Genepp à Rivière, ce sont donc les folkloristes, travaillant sur les sociétés à écriture, qui se sentent les plus concernés par la géographie humaine.

\section{Le "fait race" et les "petites foules": la sémantique de Jean Brunhes}

Dans La Géographie humaine, Brunhes est très clair sur la question des races: "Nous considérons comme n'appartenant pas à la géographie humaine l'étude des races ou des langues, étude qui repose sur des observations somatiques ou philologiques, qui n'ont rien à voir avec la géographie. ” (Brunhes 1910: 626) Dans un autre texte paru dans L'Ethnographie, Brunhes conteste l'explication déterministe de la race, affirmant par exemple que, grâce à la géographie humaine, «des faits de nomadisme pastoral [...] autrefois interprétés comme dérivant uniquement du fait race ou comme caractérisant le fait race " peuvent être reliés à "des conditions fondamentales de sol et de climat" (Brunhes 1913: 32). Au sujet des autochromes et des films des Archives de la Planète, dont le but «est d'établir comme un dossier de l'humanité prise en pleine vie, au commencement du $x x^{e}$ siècle, à l'heure critique de l'une des "mues" économiques, géographiques et historiques les plus complètes qu'on ait jamais pu constater » (ibid.: 38), il signale l'intérêt ethnographique et géographique de documenter ce qu'il nomme «les petites foules": 
[...] c'est-à-dire les groupes humains variant de cinq à quinze personnes et se trouvant représenter une forme de vie collective naturelle: hommes causant ensemble dans la rue, hommes en prière, hommes assis pour manger ou pour jouer, hommes traitant un marché dans une foire, etc. [...] Ce n'est pas lorsque les hommes paraissent immobiles que leurs mouvements sont moins intéressants; tout au contraire, c'est alors, dans ces instants de repos relatifs, que les hommes traduisent le mieux les caractères distinctifs de leur race ou de leur personne par des gestes, par des mouvements d'épaules ou de tête, par des regards. (Ibid. : 38)

Contrairement «aux musées ethnographiques » où «les types humains sont représentés par des moulages de cire ou des photographies posées ", les Archives de la Planète souhaitent, au contraire, "saisir les hommes dans la vérité même de leurs attitudes courantes» (ibid.: 39). Brunhes distingue, par ailleurs, les «petites foules homogènes", «qui sont par là des expressions typiques d'un groupe ethnique», des «petites foules hétérogènes [...] représentant par là même le mélange d'intérêts et de vie qui résulte de la juxtaposition et de la compénétration de plusieurs races, en une même région ou en un même point du globe» (ibid. : 39).

La notion originale de «petite foule » mérite qu'on s'y attarde. On sait à quel point la «foule» a hanté les esprits au XIX siècle, fascinant écrivains et artistes, obsédant études sociales et médicales. Bestiale et irrationnelle, ainsi que le suggère Gustave Le Bon dans le très influent Psychologie des foules (1895), elle effraye les élites intellectuelles. Néanmoins, la foule surgit ici sous une lumière positive. Pour Brunhes, les «petites foules» saisies «en pleine vie» (ibid. : 38) par les autochromes des Archives de la Planète constituent une alternative aux moulages de cire et aux photographies anthropométriques prisées par l'ethnographie et l'anthropologie. Cette production photocinématographique apparaît ainsi à la fois distincte et complémentaire de celle pratiquée par l'ethnographie. Distincte, dans la mesure où elle privilégie des protocoles de documentation différents: les Archives, dont «les instruments qui viennent de naître [sont employés] pour saisir et conserver les faits de la planète qui vont mourir » (ibid. : 38), capturent ces actions et ces gestes dans leur cadre et leur environnement naturels, à l'inverse des musées d'ethnographie et des herbiers, où, dit Brunhes, l'« on collectionne les plantes mortes» (ibid. : 38). Complémentaire, parce que cette «documentation vraiment moderne» permet d'«apporter une aide précieuse aux recherches ethnographiques» (ibid.: 39). Si Brunhes ne s'affranchit pas de la notion de race (ce n'est qu'après la Seconde Guerre mondiale que les classifications raciales sont globalement écartées comme non scientifiques), il est évident que l'étude biologique de la variabilité humaine et son penchant déterministe d'alors l'intéressent peu. Dans un autre texte publié en 1917, «Races et nation: les plus lointaines origines de notre peuple de France », il s'attaque à nouveau à cette notion, observant d'emblée:

Tous les savants dont les esprits ne sont pas régis par d'absurdes et dangereuses préoccupations politiques reconnaissent que le rôle attribué à la race, dans l'histoire, a été exagéré ou faussé. II n'y a pas de race civilisée qui soit pure; aucun des ensembles politiques d'aujourd'hui ne correspond à une race homogène. La race n'existe précieuse liaison avec la discipline géographique» (cité dans Segalen 2002). 
5. La «nouvelle vision» ("Das Neue Sehen") est un courant photographique moderniste né en Allemagne. Ce mouvement rejette le mode de représentation frontal et horizontal caractéristique du pictorialisme et privilégie des angles de vue inédits (plongées, contre-plongées) et/ou des cadrages rapprochés.

6. Lettre à Paul Valéry, 23 octobre 1929. Paris, bibliothèque Jacques-Doucet, VRY 298 in $-4^{\circ}$.

\section{ci-contre}

fig. 3

Jean Brunhes, Races.

Paris, Firmin-Didot, planche 95 pas, mais la nation existe, qui a donné à des peuples d'origines anthropologiques, d'appartenances linguistiques et de dépendances religieuses différentes une cohésion, voire, en certains cas, une union extraordinaire. (Brunhes 1917: 769)

Certes, l'expression «race civilisée » - et l'idée, non exclue, que des races «non civilisées » puissent demeurer «pures » - nous rappelle à quel point Brunhes est un penseur de son temps. Cela dit, il peut paraître étonnant que Brunhes ait signé en 1930 un ouvrage intitulé Races, car en tant que discipline intéressée par des groupements humains particuliers (métiers, groupes confessionnels, etc.), la géographie humaine était peu encline aux dérives de type "bio-sociologique" associées à ce concept, en particulier dans les années 1930. Mais que savons-nous sur ce livre d'images et sur la collection dans laquelle il est publié?

\section{La collection "Images du monde"}

Lancée par Jacques Haumont, (1899-1974) directeur littéraire des prestigieuses éditions Firmin-Didot, "Images du monde, encyclopédie illustrée de la vie moderne » est dirigée par Florent Fels (1891-1977), écrivain, journaliste, critique d'art et rédacteur en chef de la revue artistique L'Art vivant, où il défend ardemment la «nouvelle vision" photographique ${ }^{5}$. Fels collabore avec le grand hebdomadaire $V u$, fondé par Lucien Vogel en 1928, et crée Voilà en 1931, aux éditions Gallimard. Ces publications - où l'image n'est plus une illustration, mais le contenu même de l'information - révolutionnent le paysage visuel de la presse française; elles exploitent des techniques innovantes, telle l'héliogravure, adaptées aux rotatives et aux grands tirages. La collection «Images du monde» est symptomatique de cette "vogue actuelle pour la photographie", remarque Fels lui-même (1931: 539), et ses fascicules faisant la part belle aux photographies ont, si l'on en croit les critiques de l'époque, frappé les esprits. La Revue mondiale qualifie ainsi le premier titre de la collection, Mer, Marines, Marins paru en avril 1930, de «livre-cinéma », comme si seul le cinéma pouvait être le paradigme de ces livres d'images (Ségur 1930: 87). La préface en est signée par Paul Valéry et le livre contient quatre-vingt-seize planches, accompagnées de courtes légendes rédigées par le capitaine au long cours Georges Dupuy. À l'instar du volume de Brunhes, les clichés proviennent de sources très diverses (une vingtaine de fonds d'archives, d'agences ou de photographes), dont certains sont d'Eli Lotar et Germaine Krull, issus de la «nouvelle vision » chère à Fels. Dans une lettre adressée à Valéry, Fels insiste sur la participation de ces «poètes de la lumière»:

La Maison Firmin-Didot m'a confié le soin de diriger ses éditions artistiques et nous allons publier une série d'ouvrages en grand format, illustrés de cent photographies exécutées par des techniciens qui sont, en même temps, des gens forts sensibles, à vrai dire des espèces de poètes de la lumière. Vous en connaissez déjà, sans doute, soit que vous avez vu leurs œuvres dans L'Art vivant, où nous avons publié des photos de Sougez, Alban, Kertész, Germaine Krull, etc., et vous avez pu vous rendre compte qu'à ces objets qui méritent, en apparence, le titre de natures mortes, ces artistes sont parvenus à donner un aspect vivant ${ }^{6}$. 


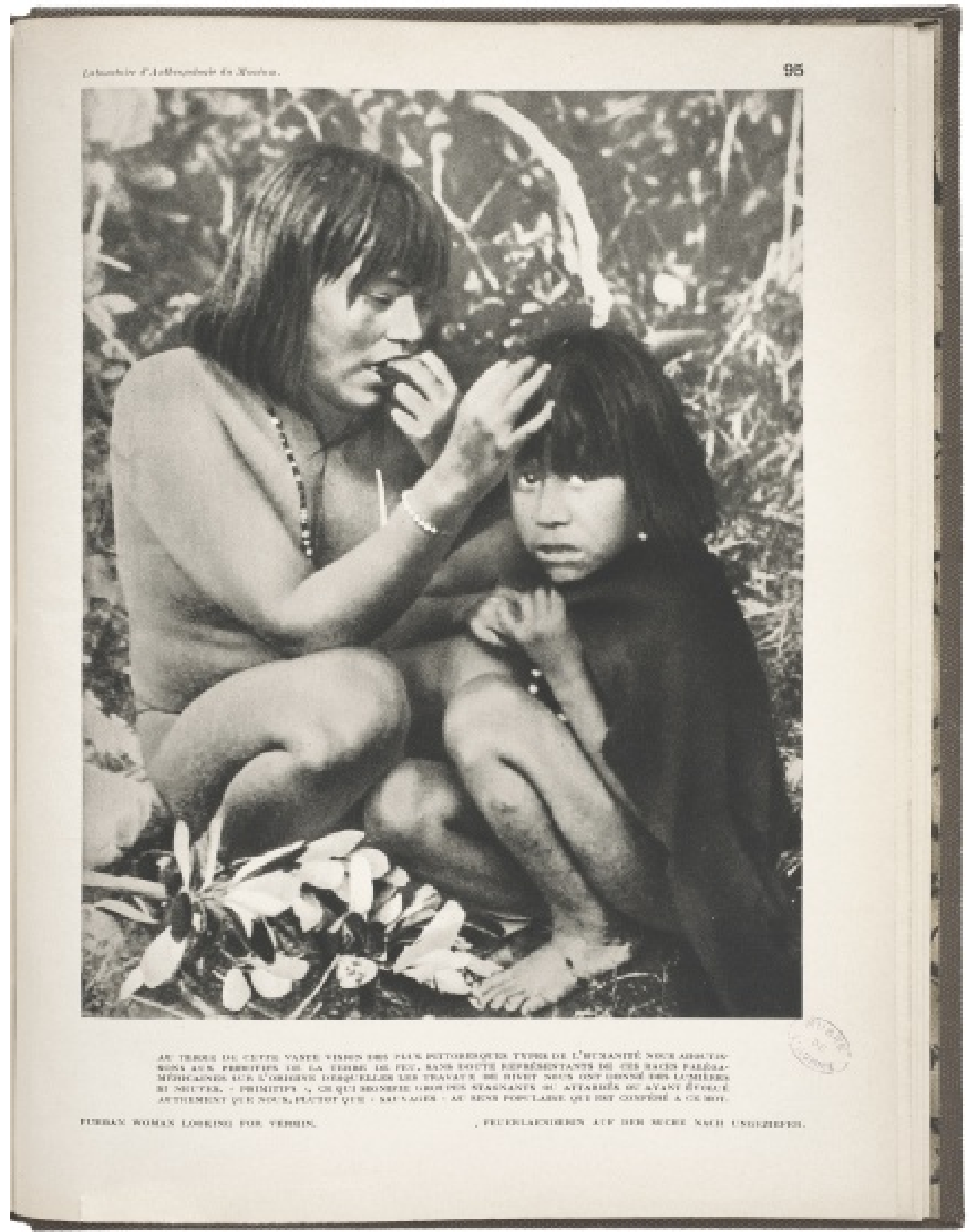




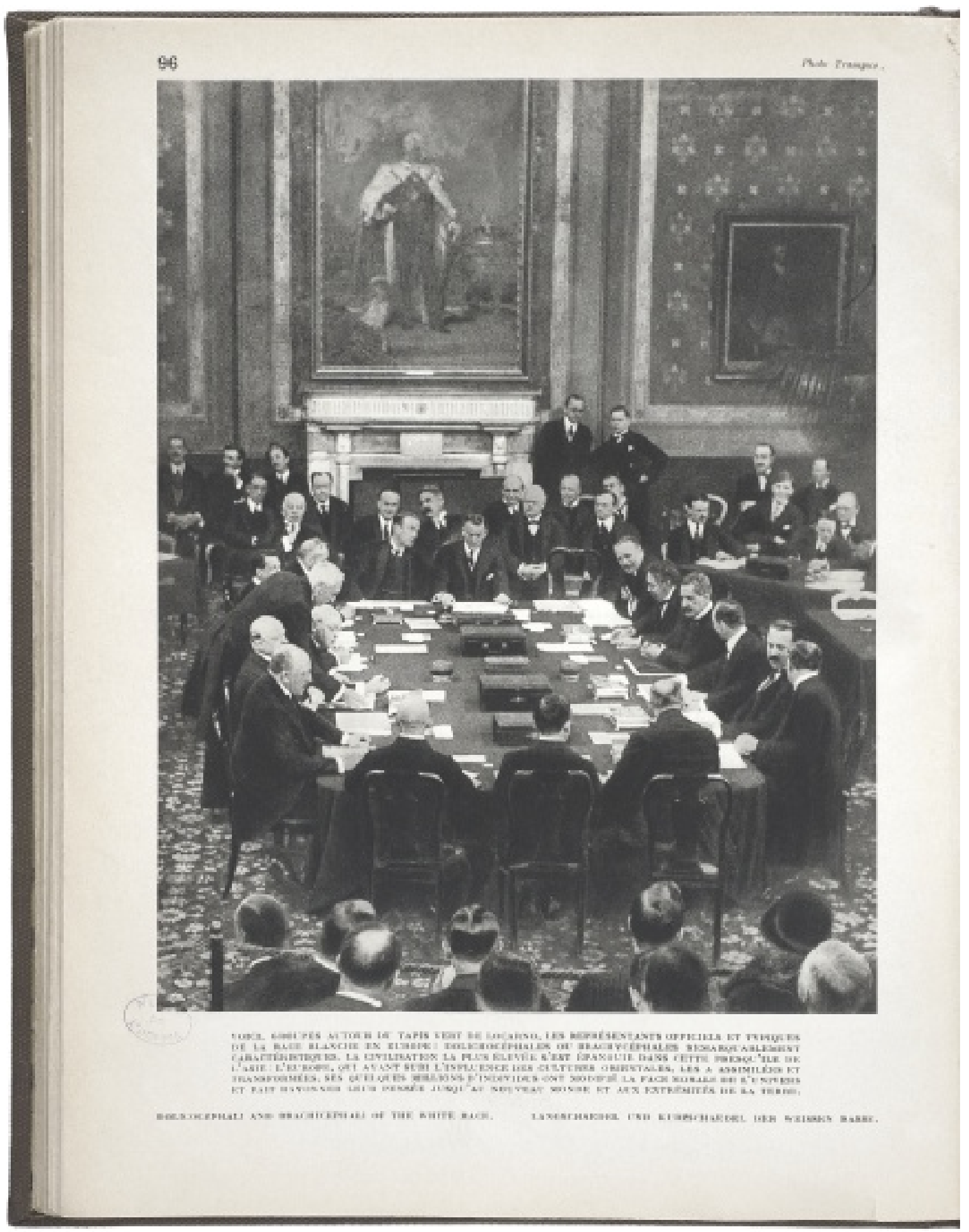


L'ouvrage réserve toutefois une place importante aux photos d'agences, preuve de l'éclectisme photographique de la collection, où seront publiés au moins cinq titres conçus sur le modèle de Mer, Marines, Marins: introduction générale confiée à un écrivain de renom, suivie de quatre-vingtseize planches tirées en héliogravure et commentées par un spécialiste ${ }^{7}$. La plaquette de présentation de la collection, ouverte à souscription et destinée au grand public ${ }^{8}$, insiste sur les documents photographiques:

II existe actuellement, dans les collections du monde entier, d'admirables documents photographiques, capables de donner de la beauté de l'univers et de l'homme un symbole émouvant. Les éditeurs ont invité celui qui a constitué la première équipe d'opérateurs véritablement artistes [Florent Fels] à réunir les plus significatifs de ces documents, à les assembler et à les mettre en valeur.

Mais il ne suffisait pas de présenter au public un simple album d'images, si belles fussent-elles. Pour donner à chacun de ces ouvrages de la collection le caractère d'utilité pratique qu'on attend de toute encyclopédie, chaque document sera commenté par une légende succincte, mais précise; la lecture de ces légendes donnera l'essentiel de ce qu'il faut savoir sur la question traitée dans chaque volume $^{9}$.

À ce premier tome viennent ainsi s'en ajouter d'autres: Races (introduction de Jean Brunhes, commentaire des images par Mariel Jean-Brunhes Delamarre, 1930, n²); Indochine (introduction d'Albert Sarraut, commentaire des images par Charles Robequin, 1930, n³), Route de Paris à la Méditerranée (introduction et documents commentés par Paul Morand, 1931, $\mathrm{n}^{\circ} 4$ ); ou encore Bêtes (introduction de Jean Giraudoux, commentaire des images par Édouard Bourdelle et Louis Roule, 1931, $\left.n^{\circ}\right)^{10}$.

Rétrospectivement, ce sont les ouvrages Route de Paris à la Méditerranée et Bêtes qui séduisent aujourd'hui l'historien de la photographie. Le premier (conçu sur une idée d'Haumont ou de Krull elle-même, alors très proche de l'éditeur) explore notamment un point de vue éminemment moderne: le paysage vu à partir d'une automobile décapotable. II compte trente-huit photographies de Krull, ainsi que des clichés d'André Kertész, Emmanuel Sougez, Moï Ver, etc. Le second, étonnant par ses suggestifs portraits d'animaux, est composé de photographies de Kertész, Krull, Jean Painlevé, etc. Pourtant, lors de sa parution en 1930, Races enchante aussi les critiques. Toujours dans les pages de Documents, Leiris vante ainsi la «poésie " du livre, cette "poésie inhérente à tout voyage " (Leiris 1930: 375) et signale «le rapprochement, fortuit peut-être mais significatif, de deux images »:

Le dernier feuillet porte, en effet, au recto: la reproduction d'une photographie prise à la Terre de Feu, sur laquelle on voit une mère fuégienne cherchant les poux dans la tête de son petit, tous les deux nus et sales - au verso: les délégués des nations européennes réunis à Locarno, "dolichocéphales ou brachycéphales remarquablement caractéristiques" en jaquette ou redingote, s'apprêtant à discuter. Beau symbole de la barbarie qui est la nôtre malgré l'hypocrisie de dehors prétentieux; beau rapprochement, en vérité moins insultant pour nous que pour les Fuégiens! (Leiris 1930: 376)
7. Selon Fels, «ces ouvrages seront précédés de quatre ou cinq pages de texte, préfacées par des écrivains à la fois célèbres et sensibles qui présenteront le thème, moins pour en commenter les variations que pour lui assigner quelques limites" (Fels 1929).

8. Le livre est vendu 30 francs, la souscription à la collection permettant aux acheteurs d'obtenir les six premiers volumes à 165 francs, payables 27,50 francs à réception de chaque ouvrage. Cette stratégie vise à capter un public pour lequel le statut de ce genre de livres n'est pas encore totalement établi, comme le suggère la remarque de Paul Reboux dans les pages de ParisSoir (toujours à propos du volume préfacé par Valéry) : "Ne trouvez-vous pas qu'un recueil de photographies qui provoque des réactions de la qualité de celle-là mérite d'être conservé dans une bibliothèque? " (Paris-Soir, 4 juin 1930, bibliothèque Jacques Doucet, VRY 298 in-4º)
9. Plaquette de présentation de la collection, bibliothèque Jacques-Doucet, VRY 298 in $-4^{\circ}$.

10. Le prospectus de présentation de la collection mentionne d'autres ouvrages dont nous n'avons pas retrouvé trace: Standard (sur la fabrication en série), Les Petites Églises de France (introduction de l'abbé Brémond, ce dernier étant aussi mentionné par Fels dans sa lettre à Valéry), La Photographie et Les Châteaux de la Loire.

\author{
ci-contre \\ fig. 4 \\ Jean Brunhes, Races. \\ Paris, Firmin-Didot, \\ planche 96.
}


L'observation de Leiris attire notre attention sur deux éléments importants du livre de Brunhes: les légendes et le montage d'images proposé. Les premières caractérisent la collection, mises d'ailleurs en avant dans la plaquette de présentation déjà mentionnée. Dans Races, c'est Mariel JeanBrunhes Delamarre, collaboratrice dévouée de son père, qui s'en charge. Voici les légendes des deux images évoquées par Leiris:

Planche 95. Au terme de cette vaste vision des plus pittoresques types de l'humanité nous aboutissons aux primitifs de la Terre de Feu, sans doute représentants de ces races paléoaméricaines sur l'origine desquelles les travaux de Rivet nous ont donné des lumières si neuves. "Primitifs», ce qui signifie groupes stagnants ou attardés ou ayant évolué autrement que nous, plutôt que "sauvages» au sens populaire qui est conféré à ce mot.

Planche 96. Voici, groupés autour du tapis vert de Locarno, les représentants officiels et typiques de la race blanche en Europe: dolichocéphales ou brachycéphales remarquablement caractéristiques. La civilisation la plus élevée s'est épanouie dans cette presqu'île de l'Asie: l'Europe, qui ayant subi l'influence des cultures orientales, les a assimilées et transformées. Ses quelques millions d'individus ont modifié la face morale de l'univers et fait rayonner leur pensée jusqu'au Nouveau Monde et aux extrémités de la Terre.

On voit bien à quel point ce montage étonnant (mais plutôt exceptionnel au sein de l'ouvrage) - ainsi que la légende de la planche 96, pouvant, par la mention des crânes dolichocéphales et brachycéphales, évoquer une sensibilité de type surréaliste - a tout pour plaire au secrétaire de rédaction de la revue Documents. Par ailleurs, la vision ouvertement évolutionniste de Mariel Jean-Brunhes y est clairement formulée. Dans son introduction, Brunhes père ne parle jamais du degré d'évolution supposé de telle ou telle «race» ni n'emploie le mot «primitifs", lui préférant l'expression «humanités rudimentaires " et mettant en garde contre des «expressions paradoxales et fautives, contradictoires même en leurs deux termes, telles que "race latine", ou "race slave" ou "race chinoise" ", ou encore contre «les principes de différenciation uniquement somatiques", qui négligent les phénomènes «spirituels et politiques» (Brunhes 1930). En effet, Brunhes semble avoir transformé l'invitation à rédiger une préface sur les «races» en une nouvelle occasion de contester la centralité de ce concept, mettant l'accent sur la nécessité d'étudier la «vie», les «gestes» et les «regards» des hommes; en ce sens, il illustre peut-être un versant plus progressiste de la «pensée raciale » propre à l'idéologie de la Troisième République (Reynaud-Paligot 2006). Avant de préciser cette dernière idée, examinons toutefois plus attentivement la sélection et le rapprochement d'images qui frappent autant l'œil de Michel Leiris. 


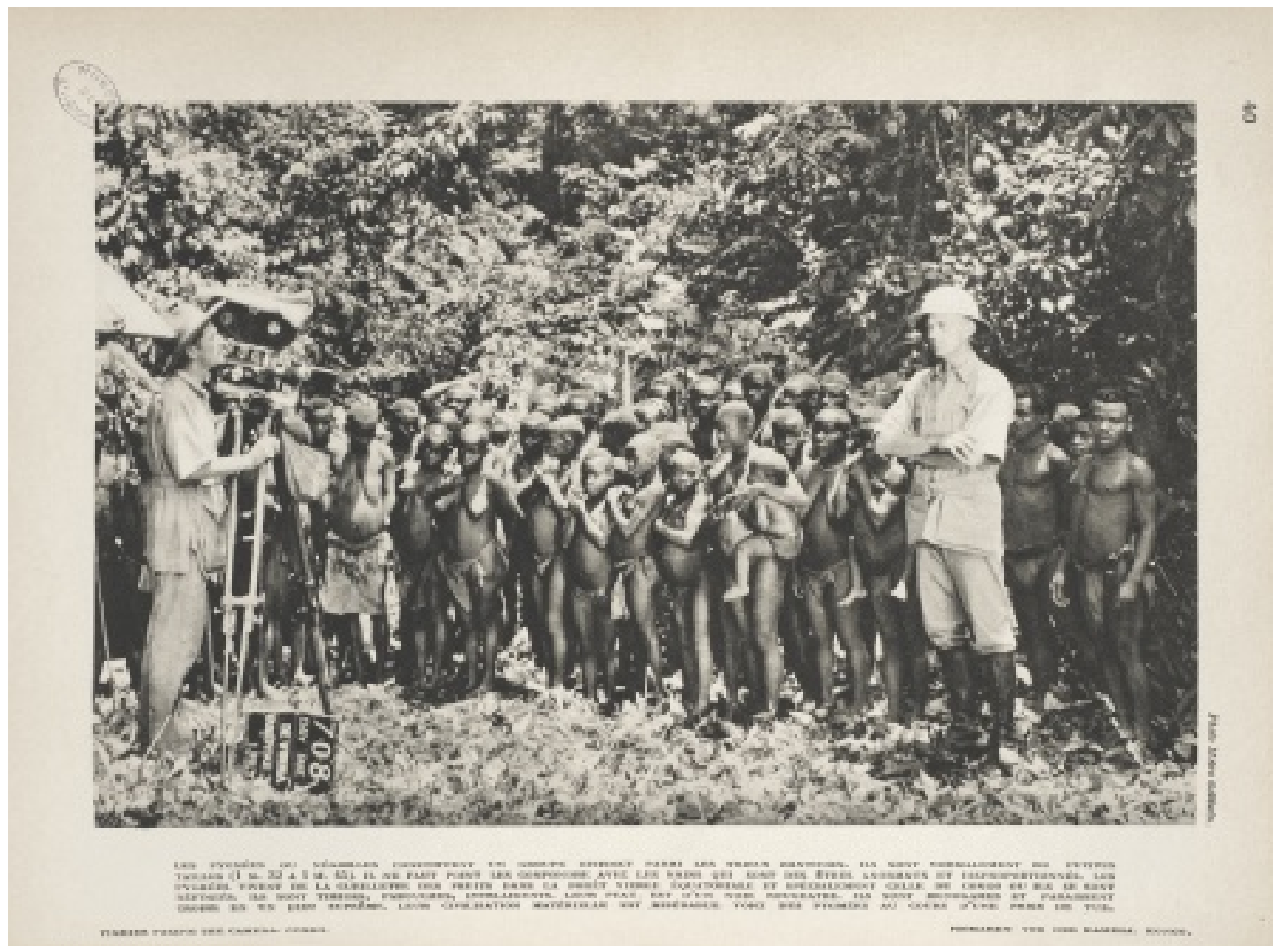

\section{Un «livre-cinéma"?}

Qui choisit les photographies reproduites dans Races? Jean Brunhes? Sa fille? Les deux (père et fille travaillant toujours en étroite collaboration)? Un(e) iconographe au service de l'éditeur? Ou Fels lui-même, comme le suggère la plaquette de présentation de la collection? En l'absence d'éléments nous permettant de répondre à ces questions, notre seule certitude est que le volume inclut des clichés de la collection personnelle de la famille Brunhes (planches 43,50 et 54 ): celle-ci est donc de toute évidence impliquée dans le travail de sélection des images. Étant donné que Mariel Jean-Brunhes est l'auteure des légendes, il est possible, par ailleurs, qu'elle ait eu son mot à dire sur l'organisation séquentielle des photographies.

L'assemblage des photographies obéit à une logique géographique, présentée par Brunhes lui-même dans son introduction: de «territoire ethnique en territoire ethnique", un itinéraire qui conduit le lecteur des «Noirs Australiens» «jusqu'au seuil de Notre Europe» (Brunhes 1930). Le livre s'ouvre ainsi sur le portrait de deux Australiens (planche 1), suivi d'images de la Mélanésie (Nouvelle-Guinée, Nouvelles-Hébrides, îles Salomon, Nouvelle-Calédonie), la Polynésie (îles Gilbert/Kiribati, îles Marquises), l'Asie du Sud-Est (Philippines, Bornéo, Bali, Java, Sumatra, îles Andaman), Madagascar, le continent africain (d'abord l'Afrique équatoriale, l'Afrique centrale et de l'Ouest puis l'Afrique du Nord), le Proche-Orient ('Égypte, Israël, la Syrie), l'Asie centrale (Turkestan, Népal), pour revenir ensuite à l'Asie du Sud-Est (Indochine), passer par la Chine et le Japon, jusqu'à la Nouvelle-Zemble, fig. 5

Jean Brunhes, Races

Paris, Firmin-Didot,

planche 40. double page suivante

fig. 6

Jean Brunhes, Races.

Paris, Firmin-Didot.

À gauche, fin de la préface

de l'auteur, à droite,

la planche 1 . 


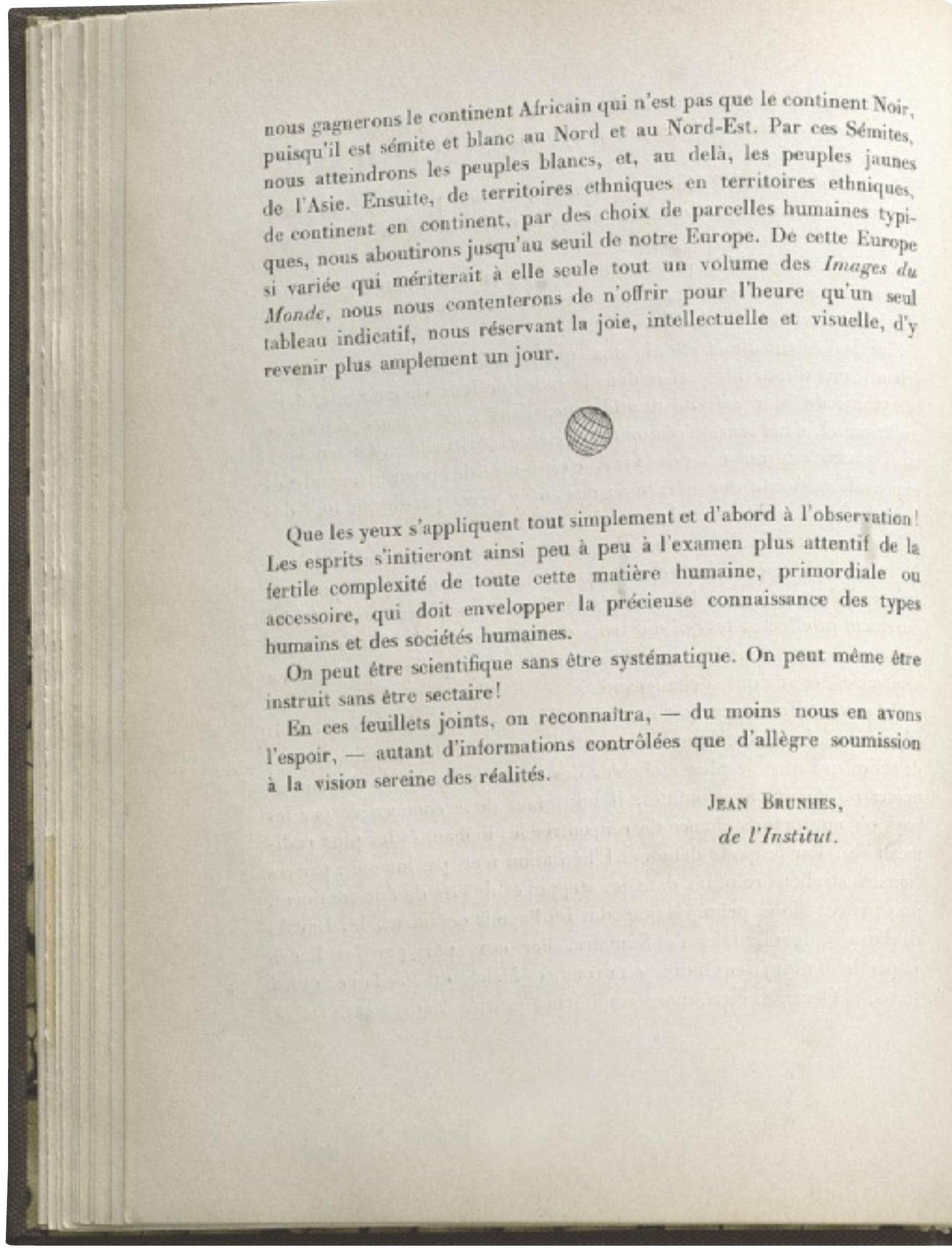



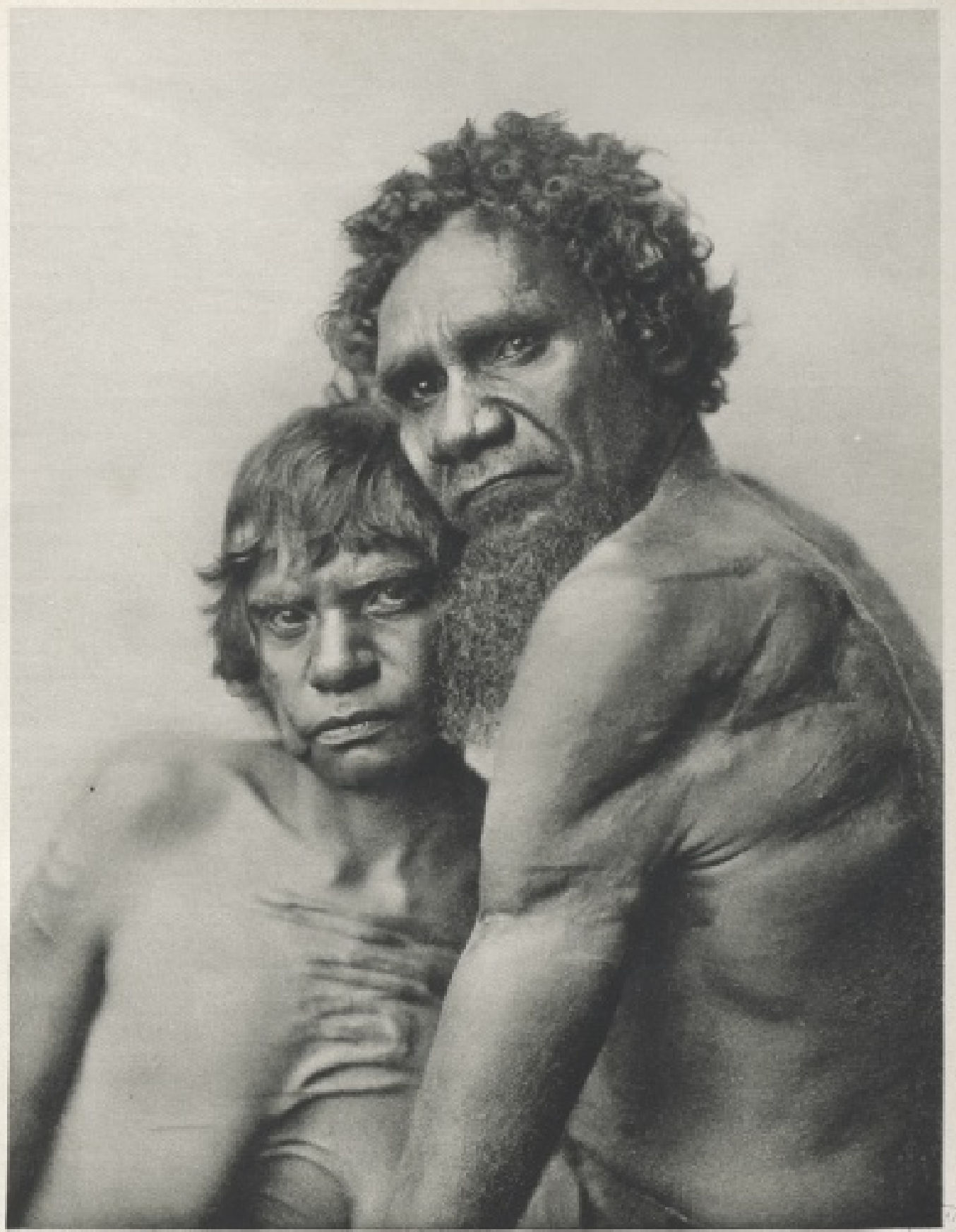

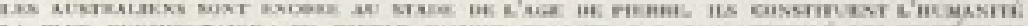

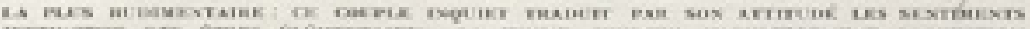

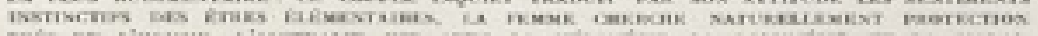

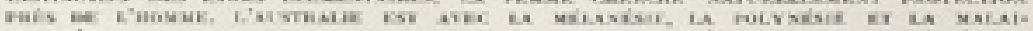

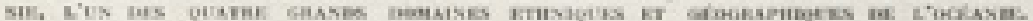


11. Voici la liste complète de ces sources:

Laboratoire

d'anthropologie du

Muséum (14 images);

Photo Forbin (23 images);

Photo Hagen (1 image);

Photo Edition Super Film

A-P. (1 image); Photo C. B.

Trautwein (1 image);

Photo Travellers' Official

Information Bureau,

Batavia (3 images)

Photo Alice-Henry

Reymond (2 images);

Photo Lapicque

(2 images); Collection

musée ethnographique

du Trocadéro (1 image)

Photo Richard (7 images);

Photo Agence économique

AOF (1 image); Photo Wide

World (5 images);

Photo Mission Citroën

(3 images); Photo MGM

(1 image); Photo UFA

Edition (1 image):

Photo Steiner (1 image);

Photo Jean et Yann

Brunhes (3 images);

Photo Rap (4 images);

Photo Agence économique

de Tunisie (3 images);

Photo Trampus (2 images);

Gouvernement général

d'Indochine (4 images);

Photo Société de

géographie (2 images):

Photo Larousse (2 images);

Photo Kikou Yamata

(1 image); Photo Laura

Adams Armer (1 image);

Photo Underwood

(1 image); Photo Collection

F. F. (1 image); Photo

O. Nordenskjöld

(4 images); Photo

d'Harcourt (1 image);

Photo Corpus missionnaire

ethnographique

Latran (1 image). aux toundras de Sibérie, au Groenland et à l'Alaska et finir sur l'Amérique du Nord (Canada, EUA, Mexique), les Caraïbes (Martinique) et l'Amérique du Sud (Guyane, Brésil, Panamá, Argentine, Pérou, Chili). Le portrait de la mère fuégienne et de son enfant ainsi que le cliché réalisé lors de la signature des accords de Locarno concluent ce vaste parcours, qui ne s'arrête jamais en Europe, la «race blanche» demeurant donc en dehors de la discussion. Brunhes écrit à ce propos: "Cette Europe si variée [...] mériterait à elle seule tout un volume des "Images du monde". " (Brunhes 1930) Cette dernière image nous rappelle, enfin, que Races constitue autant un voyage dans l'espace que dans le temps de l'humanité, le livre nous transportant « de l'âge de pierre [...]", dans lequel se trouveraient encore les «Australiens", (voir la légende de la première image du livre) jusqu'à la modernité européenne.

Les critères d'ordre chronologique semblent avoir été ignorés dans la sélection des images: les dates de réalisation des photographies ne sont jamais mentionnées, comme si leur dimension documentaire rendait transparent leur statut d'objets manufacturés. Ainsi, des clichés du XIX ${ }^{e}$ siècle (planches 18 et 83 , entre autres) coexistent avec des photographies très récentes (planche 40). Cette dernière image, créditée MGM (la célèbre société de production américaine), est en réalité une photographie de tournage de Trader Horn (Horn le trafiquant, 1931), film sonore présenté à sa sortie comme le premier film de fiction jamais tourné en Afrique et qui inspirera à la MGM le tournage de Tarzan en 1932. Le tournage, qui dure un an et demi et ne s'achève qu'en août 1930, conduit en effet l'équipe dans cinq colonies européennes, dont le Kenya, l'Ouganda et le Congo. La présence de ce cliché dans le livre de Brunhes atteste une circulation d'images foisonnante (liée, peut-être, aux multiples casquettes de Fels et à ses liens avec la presse illustrée), ainsi que la porosité des frontières entre ce qui relève du spectacle et de la science.

Les quatre-vingt-seize planches proviennent, nous l'avons dit, de sources très distinctes, les "poètes de la lumière" si chers à Fels étant cette fois absents, à l'exclusion (peut-être) d'André Steiner (planche 42). La liste en est éclairante, fournissant un aperçu du commerce et de la circulation d'images dans un paysage visuel radicalement reconfiguré par les possibilités de la reproduction mécanisée. Parmi celles-ci, on compte:

-des agences photographiques ou de presse, françaises et étrangères (Rap, Trampus, Underwood \& Underwood, Wide World, etc.);

- des collections spécialisées (le laboratoire d'anthropologie du Muséum, la Société de géographie, le musée ethnographique du Trocadéro, etc.);

- des agences économiques et/ou des institutions coloniales françaises et étrangères (Travellers' Official Information Bureau of Batavia, gouvernement général d'Indochine, Agence économique AOF, Agence économique de Tunisie);

- des maisons d'édition (Larousse);

- des collections privées françaises et étrangères, notamment de Jean Brunhes, mais aussi d'autres scientifiques (Bernhard Hagen, Louis Lapicque, Otto Nordenskjöld), journalistes (Victor Forbin), artistes et écrivains (Laura Adams Armer, Kikou Yamata), etc.;

- des missions spécifiques (mission Citroën; Corpus missionnaire ethnographique Latran);

-des sociétés cinématographiques (MGM, UFA, etc.) ${ }^{\mathbf{1 1}}$. 
Malgré les différences considérables en matière de composition et de registre, on constate l'absence, remarquable dans un volume consacré aux races, de photographies anthropométriques. II s'agit là (on y reviendra) d'un élément symptomatique de la position de Brunhes: il ne passera pas inaperçu. Mais cette collection disparate d'images se distingue également par l'attention constante portée au cadre et à l'environnement naturel typiques de la démarche de Brunhes - sans qu'on puisse pourtant y déceler les mêmes modalités iconographiques «de et à la manière» du géographe identifiées ailleurs (Mendibil 1993a et 1993b). La seule exception semble être la planche 1, qui nous montre un couple d'Australiens photographiés sur fond neutre, dans une pose rappelant certaines conventions du XIX ${ }^{\mathrm{e}}$ siècle ${ }^{\mathbf{1 2}}$. Si certaines photographies saisissent des «petites foules " (planche 17 et 97, entre autres), plusieurs portraits posés, individuels ou de groupe, scandent aussi le livre (planches 15, 75 ou 94, entre autres). Enfin, certains clichés ont été fortement retouchés (planches 23 et 79), comme il est alors de coutume. L'ensemble illustre en tout cas l'idée qu'il y a «une manière géographique de voir les faits humains sur l'épiderme de notre globe» (Brunhes 1913: 32). En somme, malgré son titre et l'hétérogénéité de ses images - ou, peut-être, justement grâce à elle -, Races témoigne d'un regard autre qu'ethnographique, malgré les indications des légendes rédigées par la fille de Brunhes: «ornements du nez [...] destinés à éviter la pénétration des mauvais esprits par la respiration » (légende de la planche 9), ou encore, pour ne citer qu'un autre exemple, le maintien de «la tradition du potlatch, cérémonie qui comprend plusieurs jours de festins et d'orgies et une distribution de richesses [...] parmi les Indiens de l'Alaska» (légende de la planche 78). L'articulation image-texte opérée par Mariel Jean-Brunhes se démarque de celle mise en place par son père, notamment dans La Géographie humaine ${ }^{\mathbf{1 3}}$. En effet, dans Races, ses légendes se limitent généralement à nommer et décrire rapidement le sujet montré, avec quelques informations supplémentaires, sans analyser, commenter ni comparer les clichés. Voici par exemple le texte accompagnant la planche 3 (le cliché d'une sculpture et d'un homme réalisé par le médecin et anthropologue allemand Berhnard Hagen en Nouvelle-Guinée, à la fin du XIX siècle): «Cette figure sculptée se dresse dans un village papou de la baie de l'Astrolabe (Nouvelle-Guinée). Toute manifestation artistique a un sens religieux ou magique chez les primitifs. Ces figurations humaines ou animales de bois ou de pierre, stylisées ou caricaturales, sont fréquentes chez les peuplades des rives du Pacifique. Ce sont non pas des dieux ou des idoles, mais plutôt des représentations de génies protecteurs et d'ancêtres. »

Revenons ici à l'assemblage des images et au rapprochement explosif entre les deux derniers clichés (95 et 96) relevé par Leiris, dont la paternité est inconnue ${ }^{\mathbf{1 4}}$. Si ce montage est très évocateur, il fait cependant exception dans l'ouvrage. En effet, au regard notamment des revues illustrées qui se multiplient, la mise en page de la collection est peu sophistiquée: les planches se succèdent simplement, comme dans de nombreux atlas d'images, les rapprochements ou écarts entre les photographies reproduites côte à côte ne semblent pas particulièrement recherchés. Ce qui compte, c'est la qualité des images et leur puissance évocatrice; pour ce qui est de leur disposition, elle semble surtout répondre à des liens thématiques ou des logiques de composition plus ou moins subtils. Ainsi, on note des effets de symétrie (planches 2-3, 24-25, etc.), de similarité de cadrage (planches 4-5, 72-73, etc.), d'opposition front/dos (planches 26-27) ou encore de résonance
12. On pourrait aussi signaler - mais ils ne constituent pas à proprement parler des images anthropométriques - les portraits de face et de profil d'une femme boubou et d'une femme sango (planches 30-31).

13. Sur l'articulation image-texte dans les différentes éditions de La Géographie Humaine, voir Mendibil 1993a.

14. II est tentant de supposer que l'idée revient à Jean Brunhes lui-même, sachant que les opérateurs des Archives de la Planète ont filmé en octobre 1925 les accords pour la paix à Locarno.

double page suivante

fig. 7

Jean Brunhes, Races.

Paris, Firmin-Didot, planches 26 et 27 . 


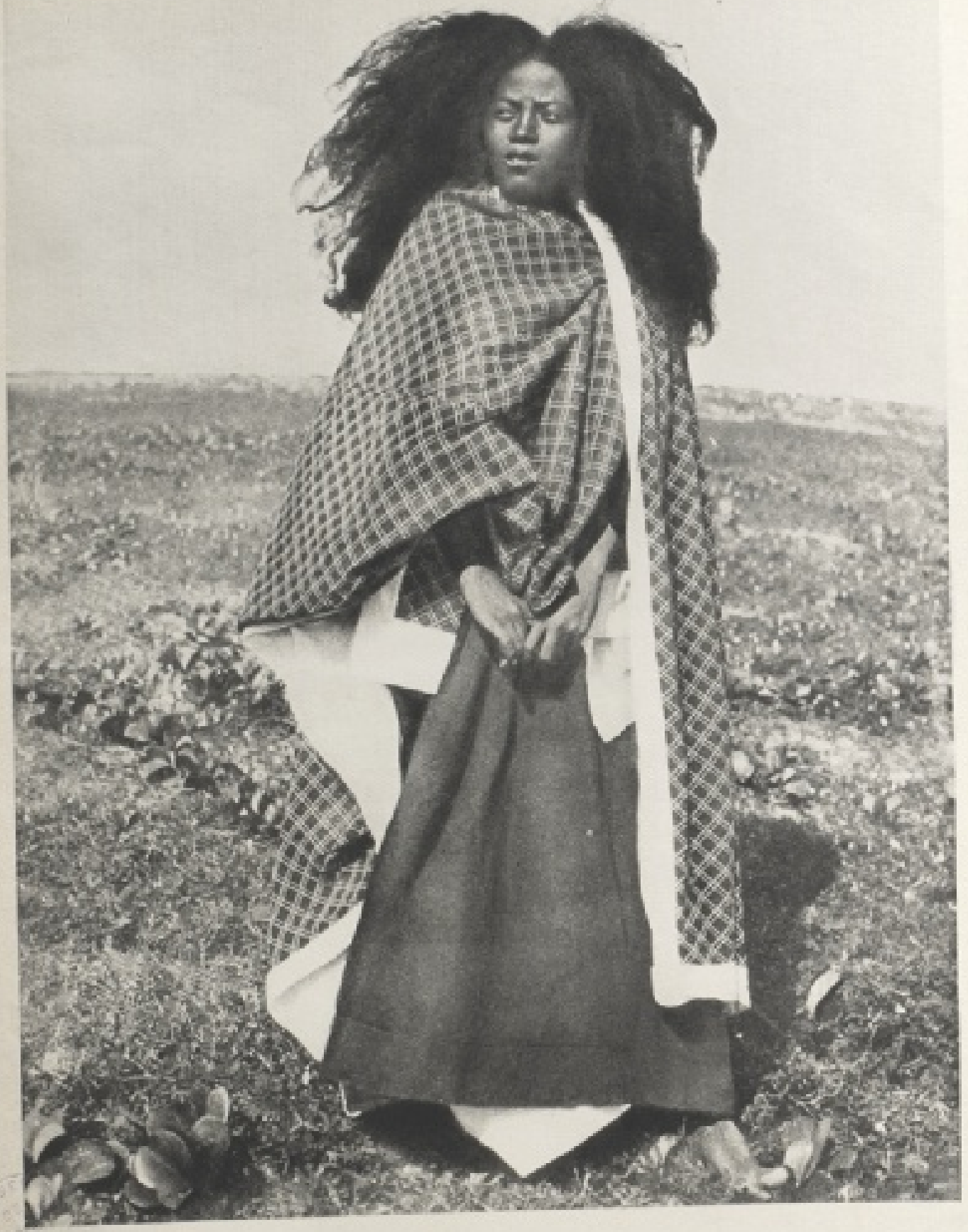

goxs navtucks

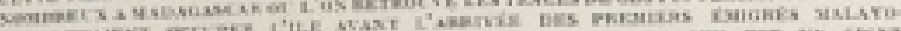
ent

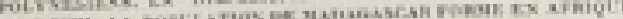

DE DEETL LA FOF 


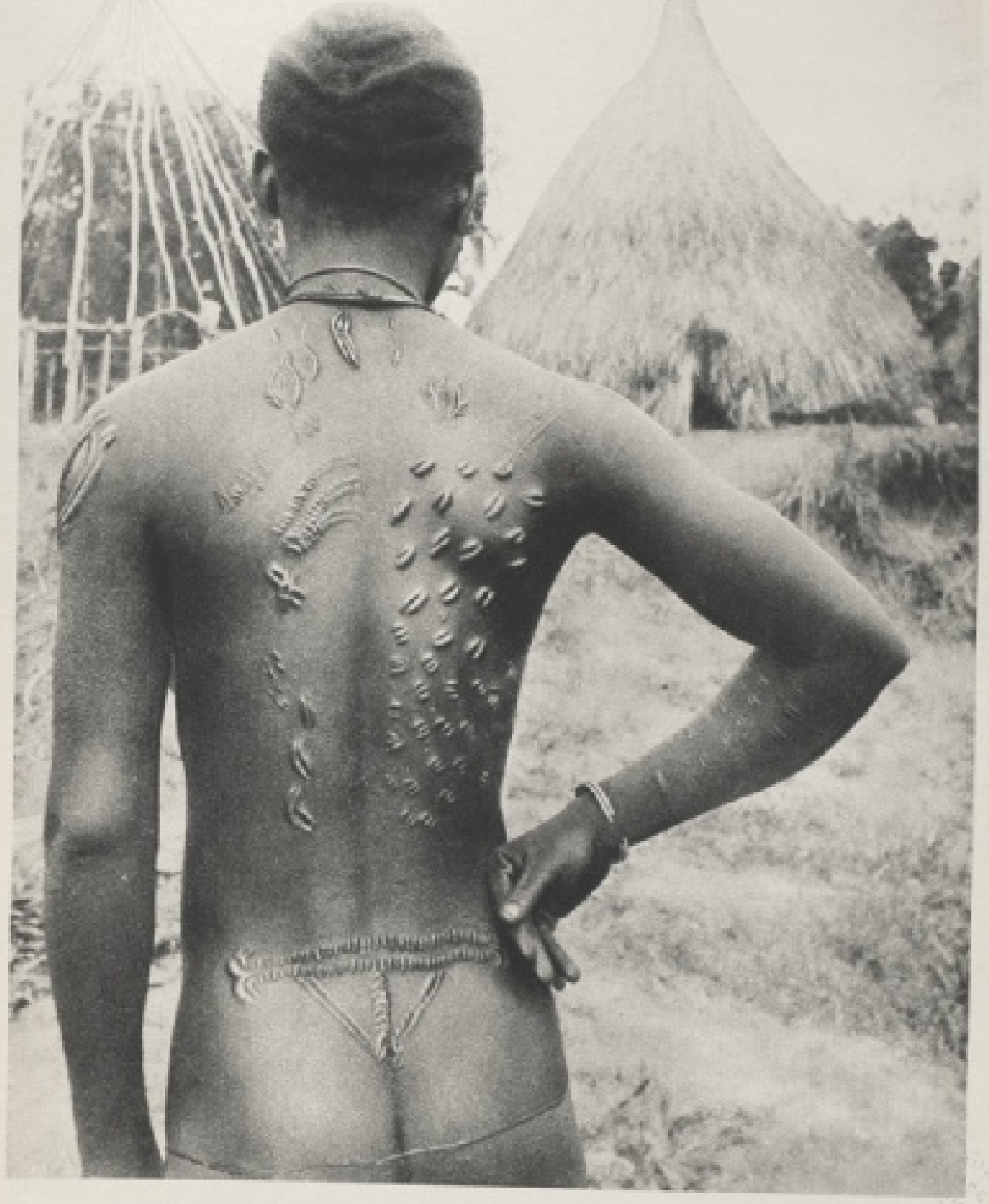

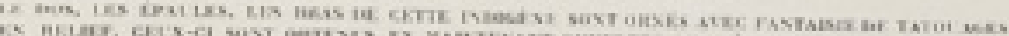

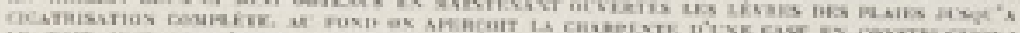

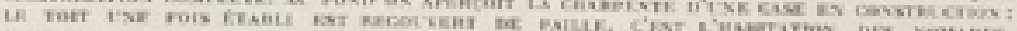

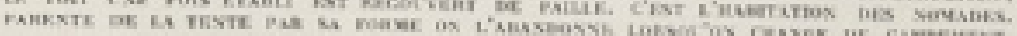


15. Voir l'exemplaire dédicacé de la main de Brunhes dans la bibliothèque du musée du quai Branly (magasin F-L-B-000384).

\section{ci-contre}

fig. 8

En haut: Jean Brunhes, Races. Paris, Firmin-Didot, planches 2 et 3 .

fig. 9

En bas: Jean Brunhes, Races. Paris, Firmin-Didot, planches 24 et 25 . des motifs (les jupes de deux femmes balinaises dans la planche 21 répondant aux habits et à l'étoffe très décorés de la planche 20). Si Races est un "livre-cinéma", ceci n'est pas dû à une utilisation inventive ou systématique du montage, au sens d'un assemblage d'hétérogénéités dont la puissance épistémologique reposerait entièrement sur les chocs et les intervalles. Les images de Races sont, certes, profondément hétérogènes sur le plan de la chronologie et du contexte de production, mais leur montage n'est pas pensé suivant le critère du choc. À l'instar de Mer, Marines, Marins, Races serait plutôt un «livre-cinéma» dans la mesure où il se fonde sur la reproduction photographique et la mise en ordre visuelle des images. Celle-ci crée des "séquences", induit une progression rythmée aussi bien dans l'espace que dans le livre et propose un véritable «voyage du regard", dont il ne faut pas négliger la portée. Au-delà de sa "poésie " (Leiris 1930: 375), il permet d'installer chez le lecteur une conscience de la réalité à partir des images. Autrement dit, ces livres ne mettent pas seulement le monde en images: ils façonnent l'image que le lecteur a du monde (et, donc, l'image qu'il a de la diversité humaine). C'est encore en cela que cet atlas de vulgarisation, s'inscrivant dans la tradition lointaine des atlas scientifiques, se rapproche du cinéma, comme le comprend bien le reporter qui, à propos de Mer, Marines, Marins, vante non seulement la portée heuristique des images en mouvement, mais leur lien souterrain avec la cartographie:

Le cinéma, que les intellectuels chargent de tous les maux, comme l'âne pelé de la fable, a eu, au moins, un bienfait: il nous a appris à regarder; il a restitué à l'œil humain son primordial privilège; grâce à lui, nous voyons le vaste monde par images, et c'est par elles que celui-ci s'incorpore à notre cerveau, ainsi qu'une nourriture spirituelle. Naguère, le monde se présentait à nous sous la forme de mots, de lignes coloriées, dans ces livres un peu secs, mais propices aux désirs et aux rêves qu'on nomme encore aujourd'hui des géographies; mais à ceux qui n'étaient pas encore doués de quelque imagination, pareils ouvrages ne disaient rien. II y avait les mappemondes, ces grosses boules dessinées de terres et baignées d'eau qui, dans la solitude d'un cabinet de travail, faisaient tourner l'univers; mais combien étaient rares les fastueux qui possédaient dans leur demeure pareil trésor! [...] Doit-on regretter le temps où l'on imaginait les choses, ou faut-il préférer le nôtre, où, pour ainsi parler, on les touche de l'œil et du doigt? (Vignaud 1930: 549)

Voir le monde par images et, qui plus est, en fonction d'un regard "géographique" introduisant des ruptures bienvenues dans les protocoles visuels très figés de l'anthropologie: voici l'enjeu de Races. Cette ambition est, néanmoins, desservie par les paradoxes du discours des Brunhes, père et fille - voire par l'ambiguïté des images elles-mêmes.

\section{Un discours - et un atlas des races - paradoxal}

Mais que propose donc Brunhes dans l'introduction de cet ouvrage, qu'il aura encore le temps de dédicacer à son ami Paul Rivet, malgré sa mort soudaine ${ }^{15}$ ? Le géographe s'inspire du nom même de la collection pour exposer le principe du livre: 

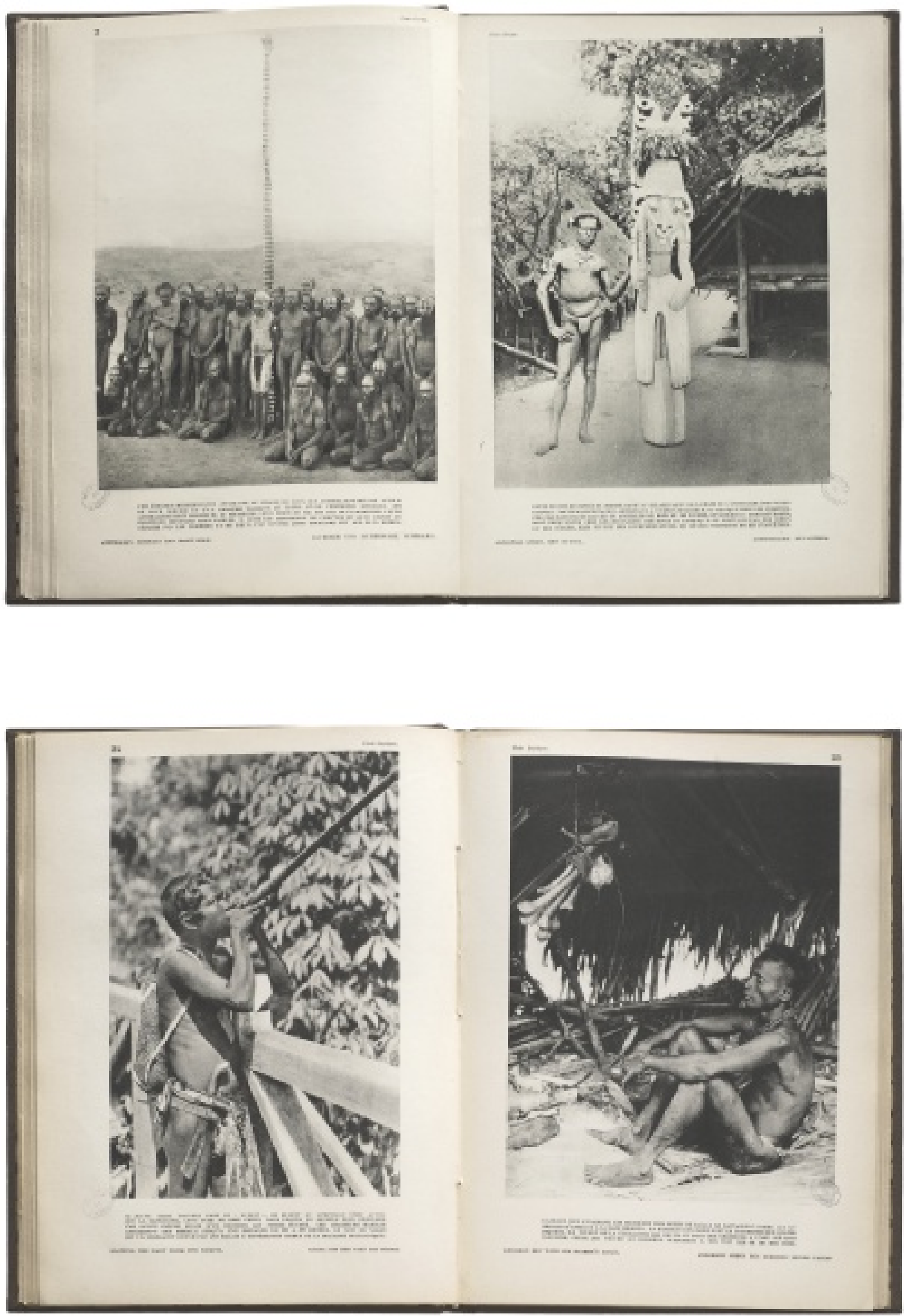

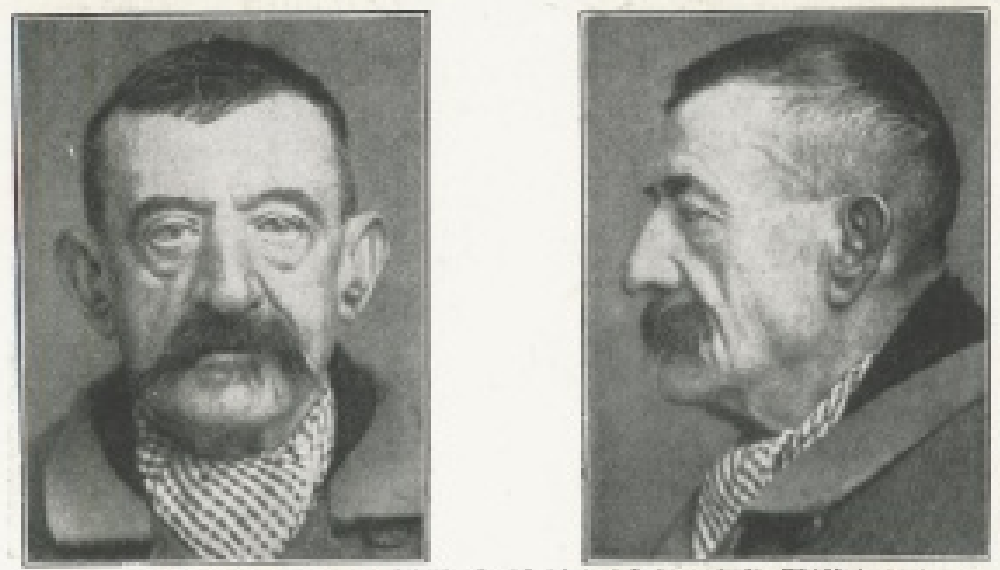

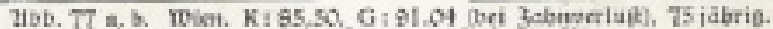
$A$ : btave mit trubblauem hiperem Ning
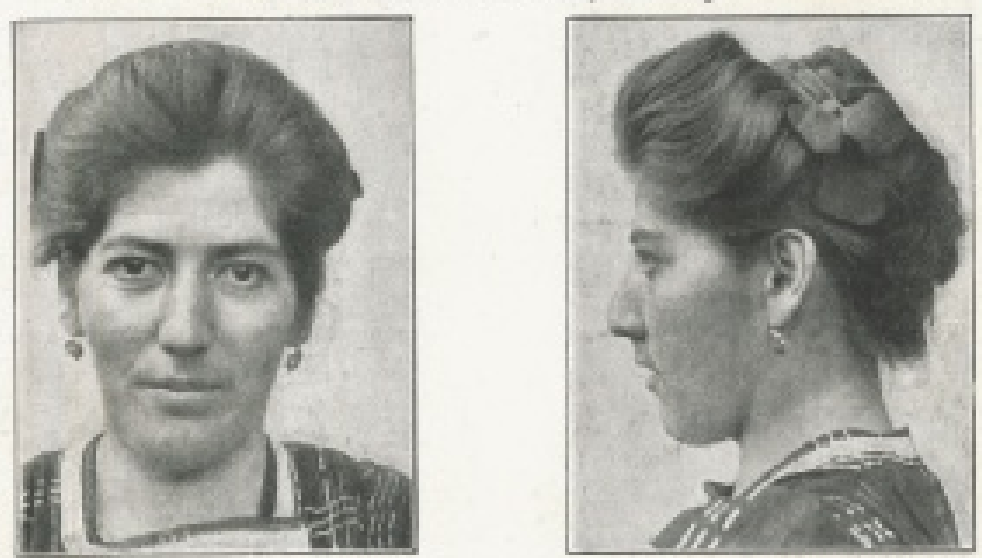

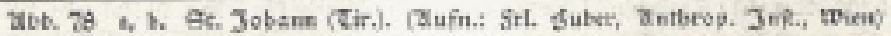
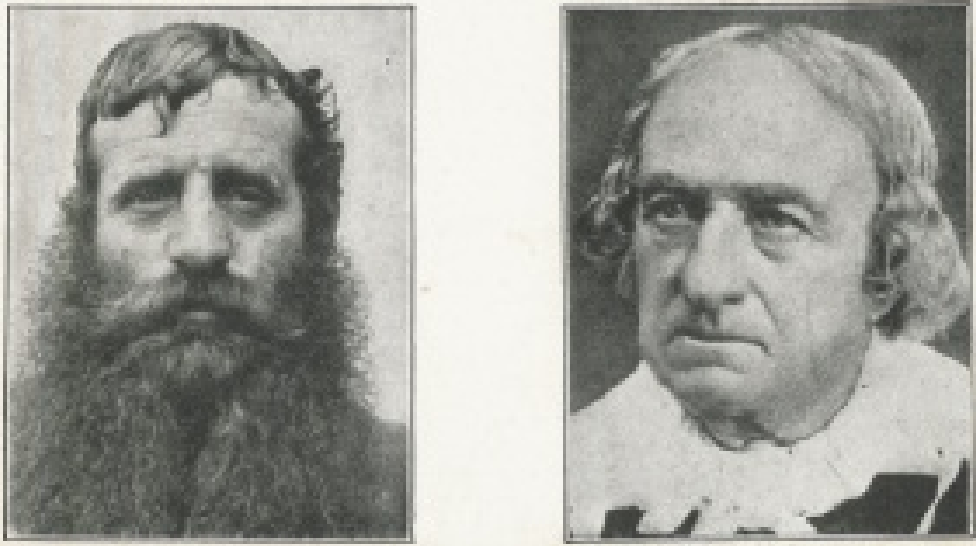

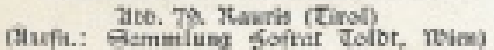

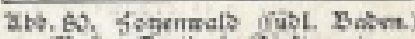

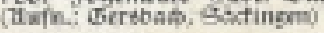
Dinarif ober porwiegend sinarif क 
Images du Monde? Mais oui : images, et non pas traité. Figures, perspectives, gestes qui doivent stimuler la curiosité sans avoir l'audace d'imposer de définitives vérités.

Accordons, sans idées préconçues, quelque regard à tant d'humanités dissemblables, qui sont pourtant bien réellement sur notre propre planète nos contemporaines!

Simples échantillons choisis aux quatre coins du monde..., mais échantillons d'individus saisis dans le cadre de leur vie courante, dans le rythme de leur activité quotidienne, dans leur "genre de vie» traditionnel, dans leurs cérémonies religieuses et rituelles... On trouvera aussi parmi ces images des types, c'est-à-dire des portraits, symboles expressifs de toute une «race»; mais on y verra par-dessus tout des groupes figurant des attitudes sociales.

Nous n'avons voulu être ni complets ni prétentieusement logiques. À l'heure de la science anthropologique où nous écrivons, qui le pourrait être, qui l'oserait, sinon les attardés confiants encore dans ces méthodes purement intellectuelles qui ont trop souvent contraint les faits à rentrer coûte que coûte dans leurs classifications habiles, quoique parfois bien arbitraires, et surtout si changeantes?

Ce modeste ouvrage doit être un tableau - trop bref certes, mais expressif - des faces, formes et manifestations de l'être humain [...]. (Brunhes 1930)

À la lecture de ces mots, il est impossible de ne pas penser aux Archives de la Planète, sorte d'atlas photo-cinématographique d'un monde en voie de disparition, répondant, malgré ses lacunes, à la nécessité de dresser l'inventaire de la planète tout entière, par le biais d'un regard embrassant (Castro 2011: 176-190). Fondées également sur des «échantillons choisis aux quatre coins du monde", ces archives sont forcément incomplètes: elles constituent, toutefois, un tableau expressif «des faces, formes et manifestations de l'être humain". Bien qu'aucune source matérielle ne permette de l'affirmer, les dix-huit ans que le géographe a consacrés aux Archives semblent avoir informé sa vision de ce livre, tout comme l'appareil iconographique des rééditions de La Géographie humaine (Mendibil 1993c). Quant à l'absence d'images provenant de la collection de Kahn dans le volume de Firmin-Didot, celle-ci peut facilement s'expliquer: I'exploitation commerciale des autochromes et des films était proscrite par le banquier ${ }^{16}$; par ailleurs, le choix de ne pas les mentionner peut être lié aux graves difficultés que traversent alors ces Archives (la crise de 1929 laisse Kahn ruiné, ses biens étant saisis en 1932).

J'aimerais attirer l'attention sur deux aspects du discours de Brunhes: la contemporanéité soulignée des phénomènes de vie documentés dans le livre et son rejet de l'anthropométrie. Sur le premier point, Brunhes est clair: «Accordons sans idées préconçues, quelque regard à tant d'humanités dissemblables, qui sont pourtant bien réellement sur notre propre planète nos contemporaines! » (Brunhes 1930)

Cet accent sur la concomitance des modes vie, caractéristique du projet des Archives de la Planète et de son envie de livrer un point de vue moral et politique, est particulièrement intéressant, dans la mesure où
16. La planche 64

(danses sacrées à Angkor, Cambodge) ressemble considérablement à celle de l'un des films de la collection de Kahn, "Danseuses à Angkor Vat ", tourné par Léon Busy à Angkor en 1921 (Al 12963). S'agirait-il d'une reproduction d'un photogramme? Le nom d'Alice-Henry Reymond, mentionné dans le crédit de l'image, est pourtant inconnu. ci-contre

fig. 10

Hans F. K. Günther, Deutsche Rassenbilder. Munich, J. F. Lehmann Verlag, 1933, planche 33. 
ci-contre

fig. 11

En haut: Jean Brunhes, Races. Paris, Firmin-Didot, planches 10 et 11 .

fig. 12

En bas: Jean Brunhes, Races. Paris, Firmin-Didot, planches 72 et 73 . les «Autres» ne sont pas placés hors du temps. Autrement dit, il n'y a pas chez Brunhes de déni de cotemporalité, ou d'allochronisme, cette «tendance persistante et systématique à placer le(s) référent(s) de l'anthropologie dans un temps autre que le présent du producteur du discours anthropologique » (Fabian 2006: 72). Telle que la conçoit Brunhes, la géographie humaine n'est pas, en effet, une science «portant sur d'autres hommes, dans un autre temps» (ibid. : 233). D'ailleurs, et malgré le topos de l'urgence commun à l'anthropologie, l'ethnographie et les Archives de la Planète, la façon dont ces dernières conçoivent leur mission - documenter l'état de sociétés sur le point de disparaître face à l'expansion que l'on croit inexorable de la civilisation dite occidentale - est singulière. Au-delà de quelques exceptions bien connues - comme les films et les autochromes réalisés en 1930 par le père Aupiais et l'opérateur Frédéric Gadmer au Bénin (Beausoleil [dir.] 1996) -, les Archives s'intéressent très peu aux sociétés sans écriture, leurs images documentant le plus souvent - y compris au Bénin - un monde où tradition et modernité se mêlent tant bien que mal.

On aurait pourtant tort de croire que cet accent sur la contemporanéité des phénomènes s'accompagne, dans Races, d'un rejet de l'évolutionnisme et de sa vision téléologique. Malgré les précautions de Brunhes dans son introduction, les légendes des images, rédigées certes par sa fille, insistent à plusieurs reprises sur l'idée que ces Autres constituent bien la trace vivante d'un autre temps - même si celui-ci «[disparaît] [...] au contact d'un autre rythme de vie» (légende de la planche 14). Si les Australiens de la planche 1 se trouvent «au stade de l'âge de pierre », la jeune Philippine de la planche 15 est ainsi décrite comme «demi-civilisée». De façon encore plus symptomatique, l'une des deux photographies de la main de Brunhes reproduites dans le volume (planche 43) fait ressortir, par son cadrage très particulier, la continuité entre les «représentants de la race nubienne» actuels et leurs «ancêtres [...] d'il y a trois mille ans", dont les profils sont sculptés sur les colosses d'Abou Simbel. En somme, bien qu'il n'y soit jamais question de «races inférieures ", de «mentalités » ou de traits héréditaires et irréductibles - et cela mérite d'être souligné -, le discours de Races demeure, en particulier à nos yeux contemporains, profondément paradoxal, comme le démontre, par ailleurs, son rapport à l'anthropologie physique. En effet, si Brunhes en critique vivement les méthodes, dont l'anthropométrie, il continue toutefois d'avoir recours à la notion de «type » et à défendre l'existence de trois races «zoologiques» : la blanche, la noire et la jaune (Brunhes 1930). Pourtant, en lisant son introduction, on comprend à nouveau que ce n'est pas le concept de race qui intéresse véritablement le géographe, mais celui de peuple ou de nation. II prend position, nous l'avons dit plus haut, contre «les principes de différenciation uniquement somatiques», qui négligent les phénomènes «spirituels et politiques», consacrant aux notions de «nationalité », «nation » et «collectivité nationale» plusieurs pages de son introduction.

Dans Races, il n'y a pas, répétons-le, de portraits anthropométriques. Cette absence remarquable semble être l'une des raisons du succès critique du livre, comme l'atteste une recension parue dans la Revue des lectures, qui revient sur l'introduction du géographe: 

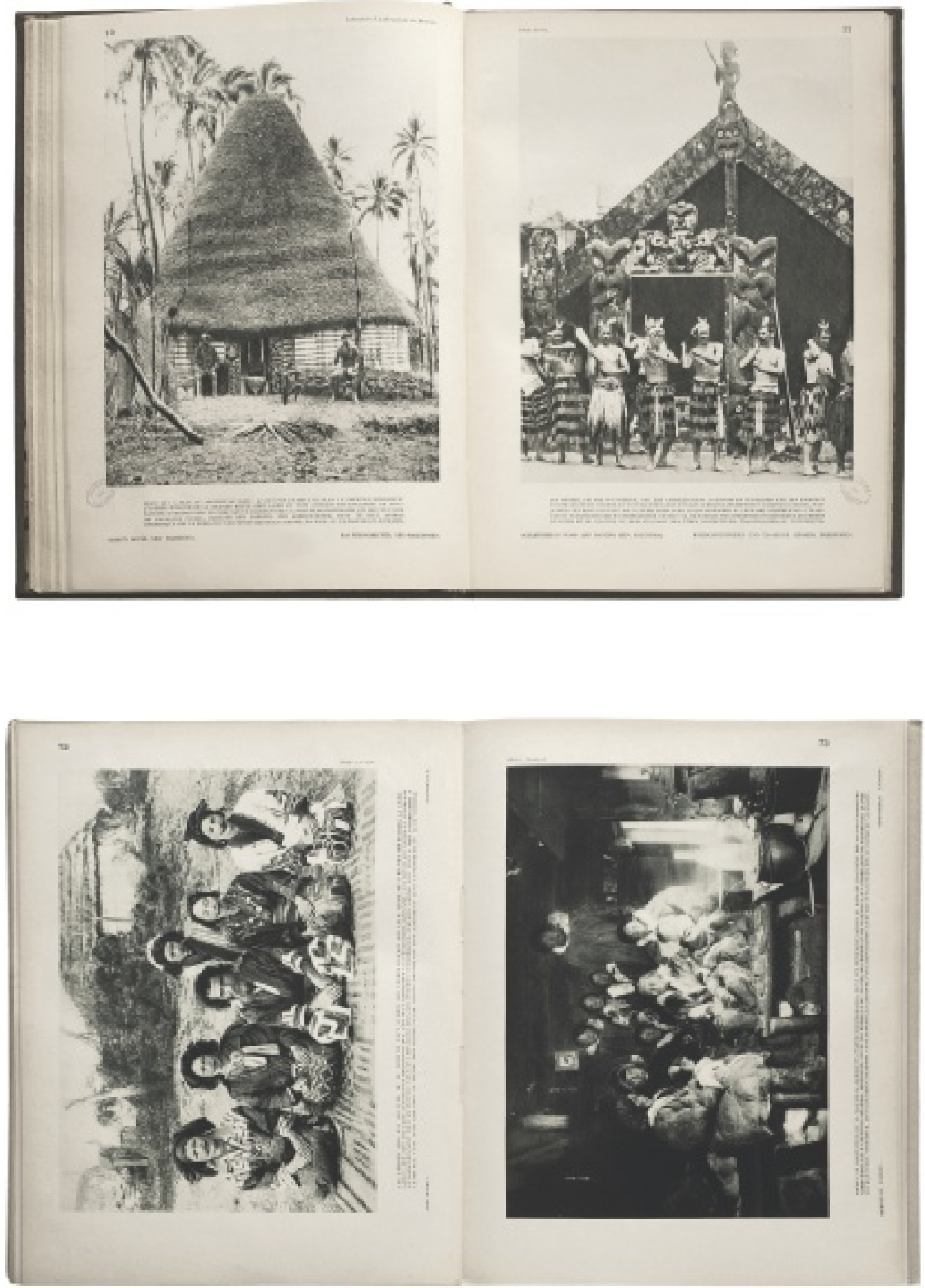


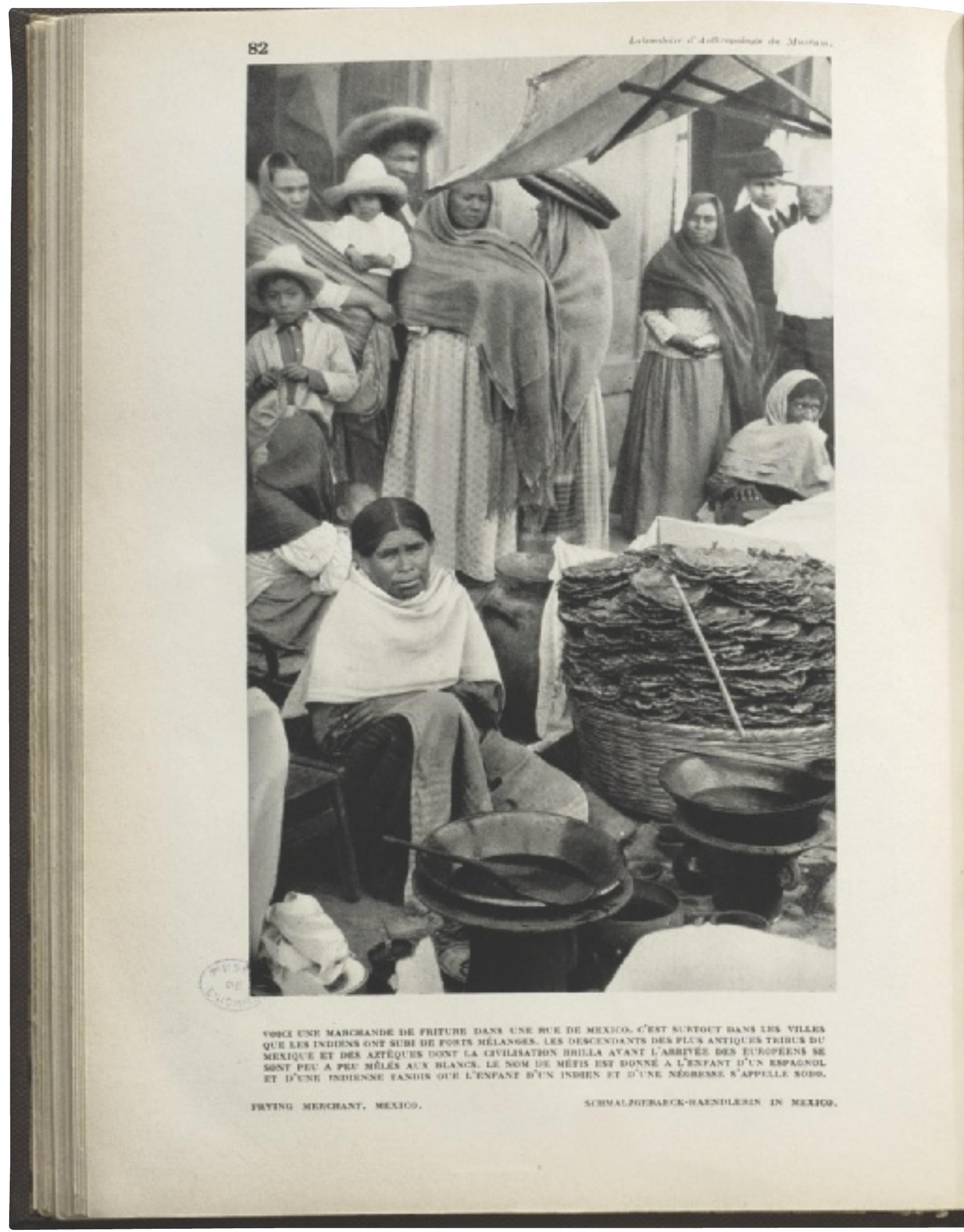




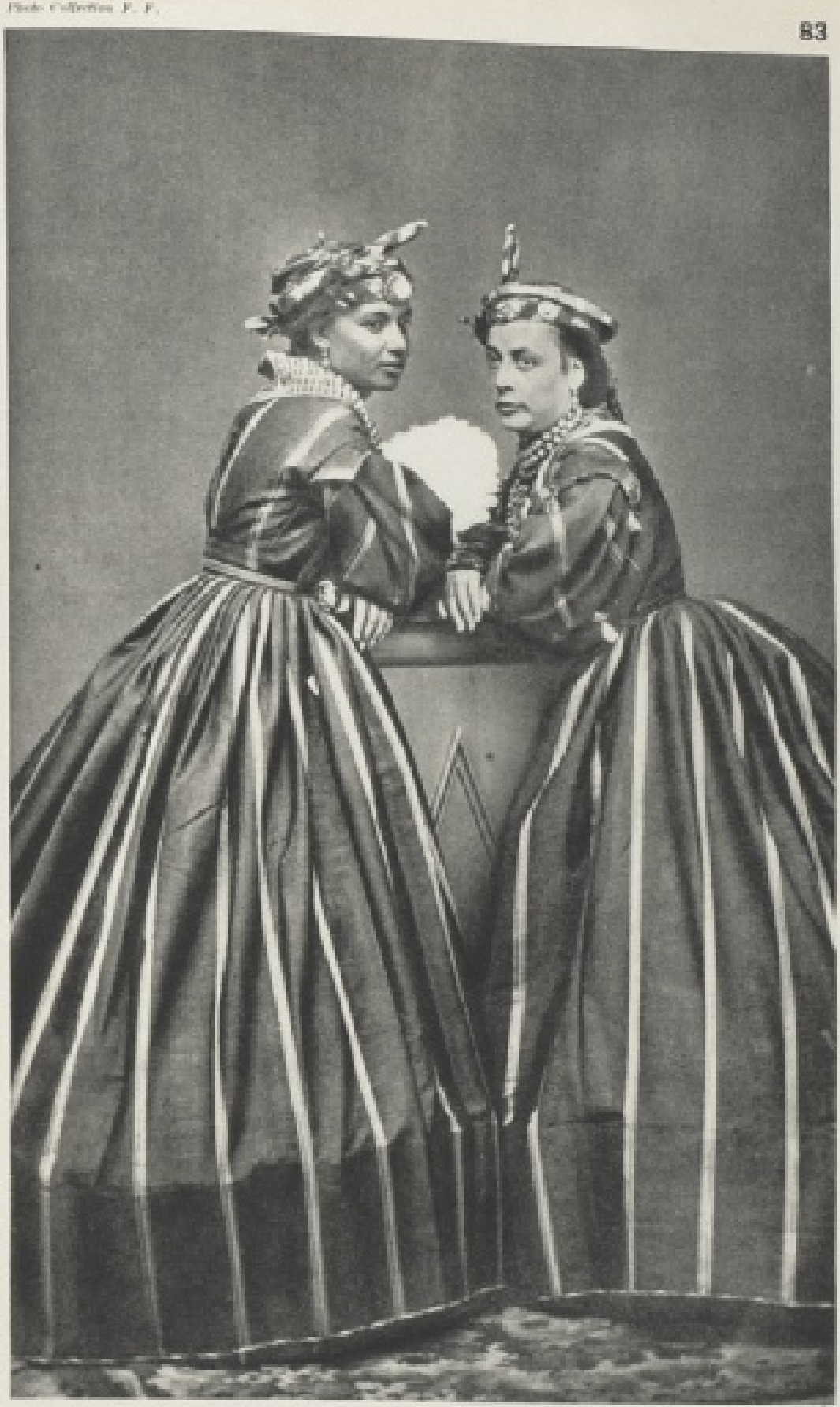

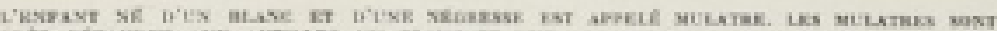

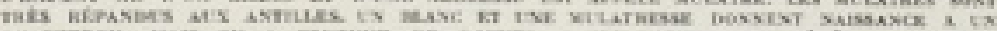

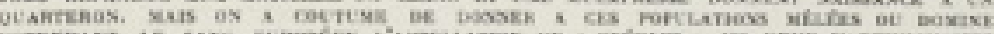

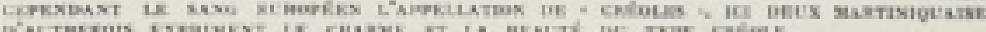


Seize pages seulement, mais si hautes et si lumineuses! Elles compteront parmi les plus belles qui soient sorties de cette noble plume. De plus, elles marquent une date dans l'histoire des sciences anthropologiques, une date décisive. Jean Brunhes y proclame la faillite de cette anthropologie matérielle qui prétendait baser ses conclusions principales sur la répartition des hommes d'après la taille, l'indice céphalique, l'angle facial, la forme du nez et la couleur des yeux. (Anonyme 1930: 1368)

De toute évidence, et malgré le discrédit progressif de l'anthropométrie dans le domaine ethnographique, la discipline, en 1930, est encore associée à cette démarche. D'ailleurs, même si ses protocoles stricts sont progressivement abandonnés dès la fin du XIX $x^{\mathrm{e}}$ siècle, l'anthropométrie laisse une empreinte considérable sur la façon de concevoir la photographie ethnographique - et en particulier les portraits, systématiquement pris de face et de profil jusqu'à des dates très tardives. Si Races n'apporte pas de solutions nouvelles à la photographique ethnographique - combinant plutôt des images hétérogènes, produites dans des circonstances et à des moments différents -, la façon enthousiaste dont l'ouvrage est reçu est peut-être symptomatique du discrédit de l'anthropométrie. Dans un contexte visuel marqué par la prolifération d'atlas de vulgarisation à caractère ouvertement raciste, en particulier en Allemagne (mais pas seulement), la déconsidération de l'anthropométrie prend aussi un caractère politique - et fait apparaître Races sous une autre lumière. Mais cela est une autre histoire.

Université Sorbonne Nouvelle - Paris III Institut de recherche sur le cinéma et l'audiovisue teresa.castro@sorbonne-nouvelle.fr 


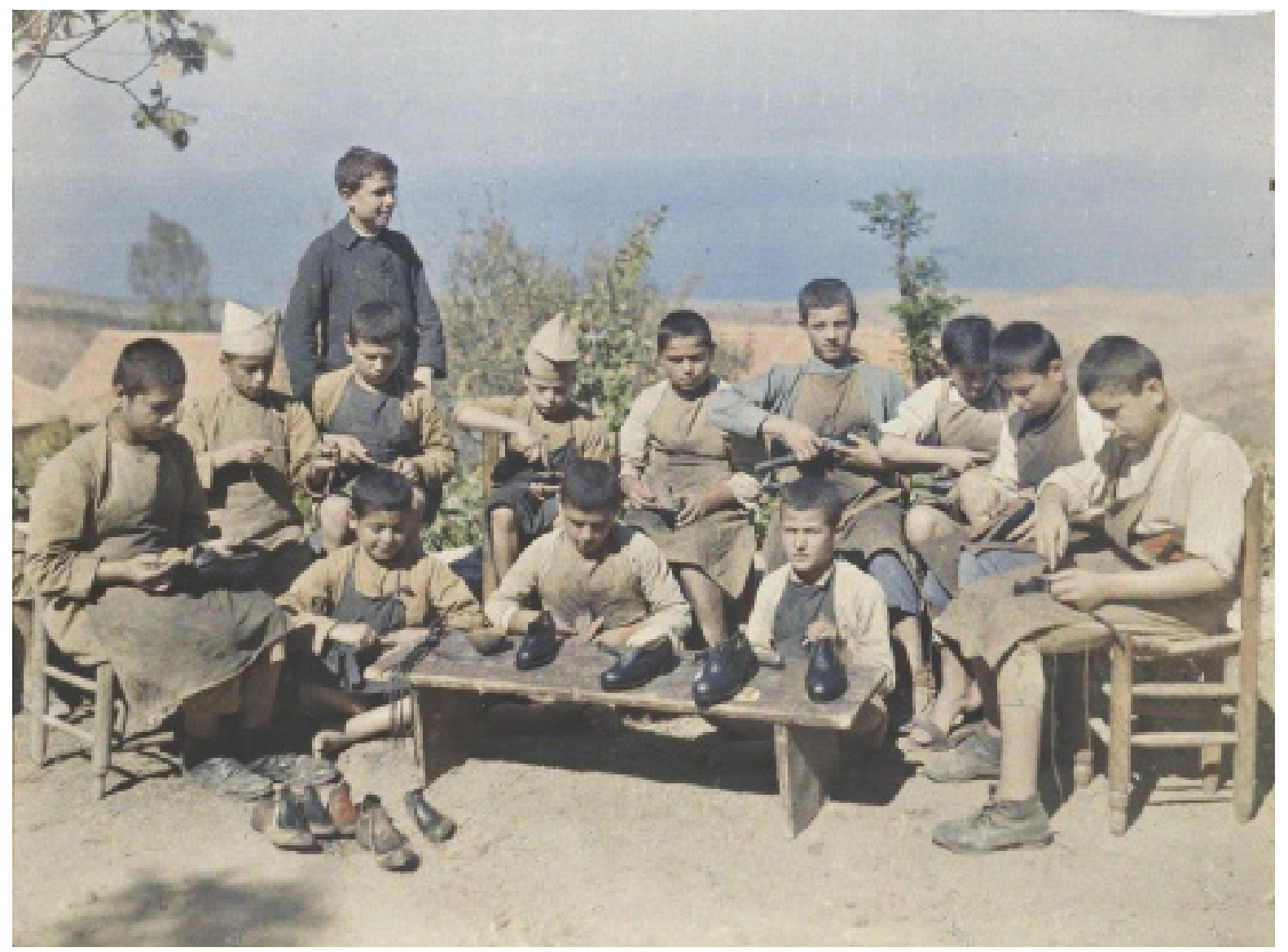

fig. 14

Frédéric Gadmer,

École industrielle française,

enfants apprenant la

cordonnerie, Liban, Aley,

7 octobre 1921. Mission

Jean Brunhes et Frédéric

Gadmer au Liban et

en Syrie, 1921.

Boulogne-Billancourt, musée départemental Albert Kahn, collection Archives de la Planète, inv. A 29271. 


\section{Anonyme}

1930 «Principales nouveautés $X$. Géographie, voyages, colonisation, missions", Revue des lectures 7 : 1368.

\section{Beausoleil, Jeanne (dir.)}

1996 Pour une reconnaissance africaine, Dahomey 1930.

Des images au service d'une idée: Albert Kahn, 1860-1940 et le père Aupiais, 1877-1945, catalogue d'exposition. Boulogne-Billancourt, musée Albert-Kahn.

\section{Blanckaert, Claude}

2004 " Géographie et anthropologie: une rencontre nécessaire (XVIII ${ }^{e}-\mathrm{xIX}^{\mathrm{e}}$ siècle) ", Ethnologie française 34 (4): 661-669.

\section{Brunhes, Jean}

1910 La Géographie humaine: essai de classification positive, principes et exemples.

Paris, Félix Alcan.

1913 "Ethnographie et géographie humaine ", L'Ethnographie $1:$ 29-40.

1917 «Races et nation: les plus lointaines origines de notre peuple de France ", Le Correspondant. Revue mensuelle : religion, philosophie, politique, 10 septembre: $769-804$

1930 Races. Paris, Firmin-Didot.

\section{Castro, Teresa}

2011 La Pensée cartographique des images: cinéma et culture visuelle. Lyon, Aléas.

\section{Deprest, Florence}

2017 «Le Collège de France en situation coloniale? Autour de quelques chaires (fin $x_{1} x^{e}$-début $x x^{e}$ siècle) ", in Wolf Feuerhahn (dir.), La Politique des chaires au Collège de France. Paris, Les Belles Lettres: 197-221.

\section{Fabian, Johannes}

2006 Le Temps et les autres: comment l'anthropologie construit son objet, trad. de l'anglais par Estelle Henry-Bossoney et Bernard Müller. Toulouse, Anarchasis.

\section{Fels, Florent}

1931 «Une Exposition de photographie», Vu 161: 539

\section{Jean-Brunhes Delamarre, Mariel}

1968 «Géographie humaine et ethnologie ", in Jean Poirier (dir.), Ethnologie générale. Paris,

Encyclopédie de la Pléiade 1465-1503.

\section{Leiris, Michel}

1930 «Compte-rendu de Races de Jean Brunhes ", Documents $6: 375-376$.

\section{Leroi-Gourhan, André}

1948 «Ethnologie et géographie humaine", Revue de géographie humaine et d'ethnologie 1: 14-19.

\section{Mauss, Marcel}

1906 «Essai sur les variations saisonnières des sociétés Eskimos. Étude de morphologie sociale", L'Année sociologique 9: 39-132.

\section{Mendibil, Didier,}

1993a « Jean Brunhes, photographe iconographe", in Jean Brunhes. Autour du monde: regard d'un géographe, regards de la géographie, catalogue d'exposition. Boulogne-Billancourt/ Paris, Musée Albert-Kahn/Vilo: 152-157.

\section{3b "Deux "manières" : Jean Brunhes et Paul Vidal de La Blache », ibid. : 52-65.}

1993c "De l'influence des Archives de la Planète sur La Géographie humaine de Jean Brunhes ", ibid. : 158-163.

\section{Orain, Olivier et Robic, Marie-Claire}

2017 "La géographie au Collège de France (milieu $x I x^{e}-$ milieu $x x^{e}$ siècle). Les aléas d'une inscription disciplinaire ", in Wolf Feuerhahn (dir.), La Politique des chaires au Collège de France. Paris, Les Belles Lettres: 435-480.

\section{Reynaud-Paligot, Carole}

2006 La République raciale: paradigme racial et idéologie républicaine (1860-1930). Paris, Presses universitaires de France.

\section{Robic, Marie-Claire}

1993a «La géographie dans le mouvement scientifique ", in Jean Brunhes. Autour du monde: regard d'un géographe, regards de la géographie, catalogue d'exposition. Boulogne-Billancourt/Paris, Musée Albert-Kahn/Vilo: 52-65.

1993b «Jean Brunhes,

un "géo-photo-graphe"expert aux Archives de la Planète ", ibid. : 109-137.

2004 "Rencontres et voisinages de deux disciplines ", Ethnologie française 34 (4) : 581-590.

2014 "La réception de Friedrich Ratzel en France et ses usages au temps de l'installation de la géographie à l'Université (années 1880-1914) ", Revue germanique internationale 20: 13-39.

\section{Segalen, Martine}

2002 «Mariel Jean-Brunhes Delamarre (1905-2001). Une œuvre entre géographie et ethnologie", Ethnologie française 32 (3) : 529-539.

\section{Ségur, Nicolas}

1930 "La vie littéraire»,

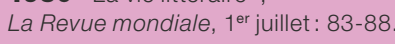

\section{Sion, Jules}

1937, "Géographie et ethnologie», Annales de géographie 263: 461.

\section{Sorre, Max}

1957 Rencontres de la géographie et de la sociologie. Paris,

Marcel Rivière.

\section{Vignaud, Jean}

1930 "Visions de mer et de Bretagne ", Miroirs du Monde, 28 juin: 549-550. 
S A.

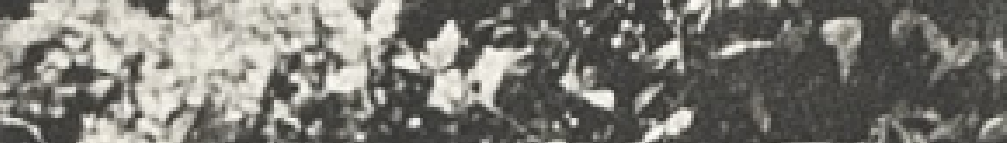

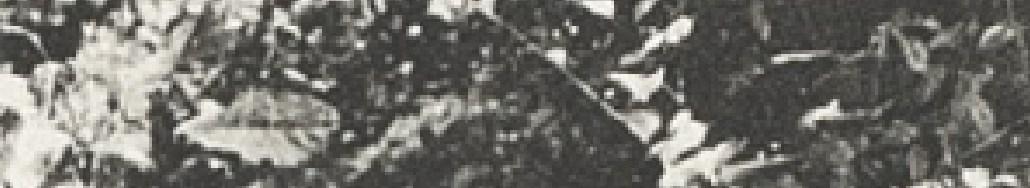

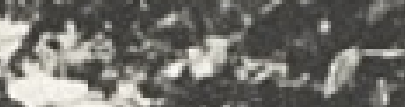

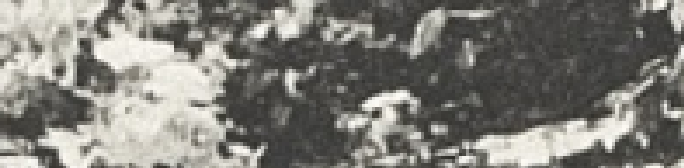
4. 2.

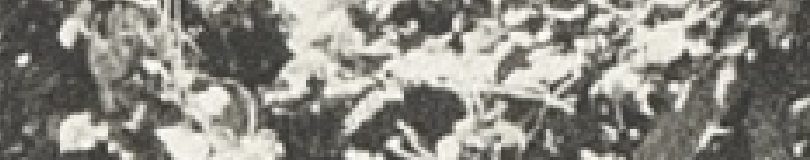

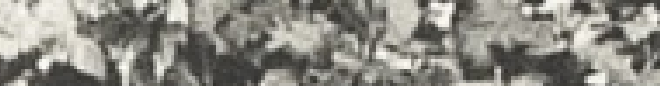

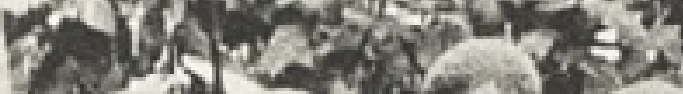

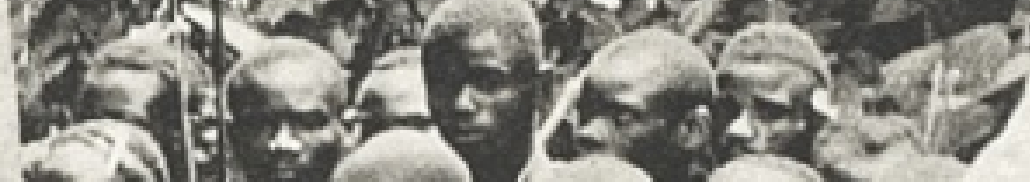

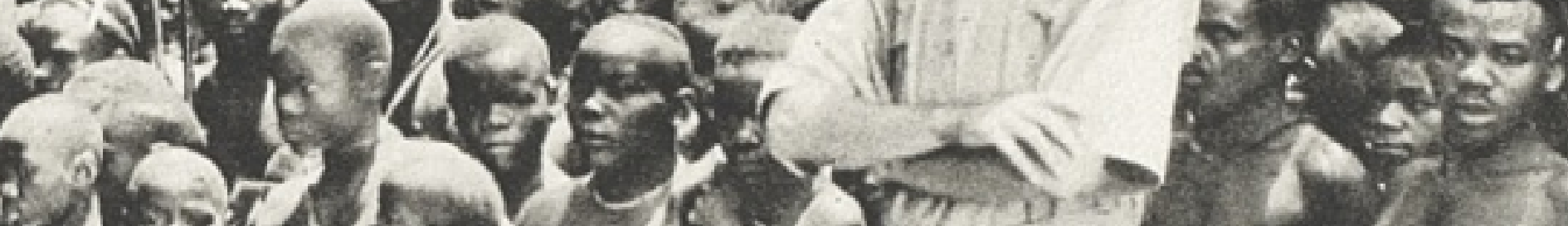

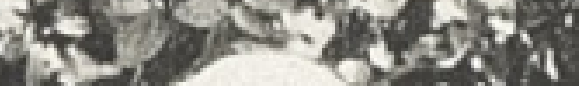

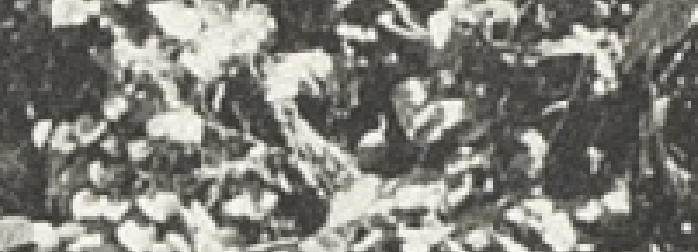

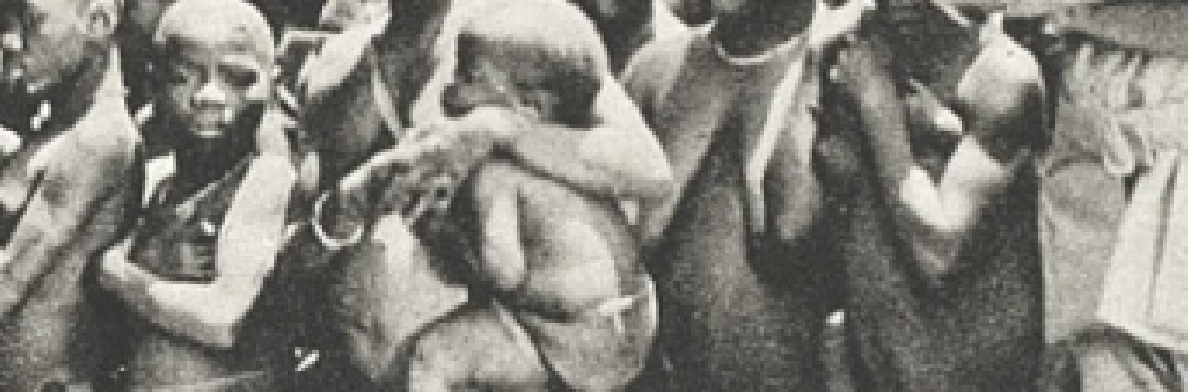

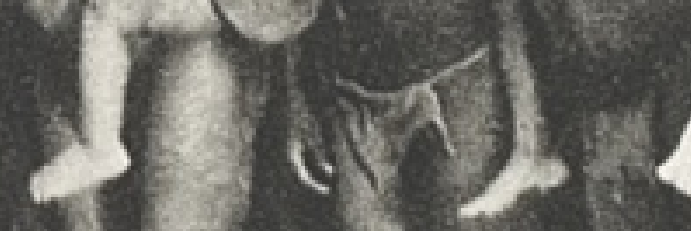
$x^{2}+2, y$

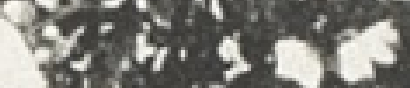

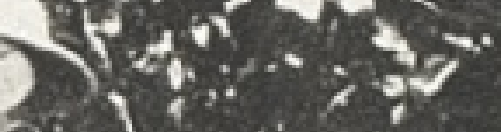

